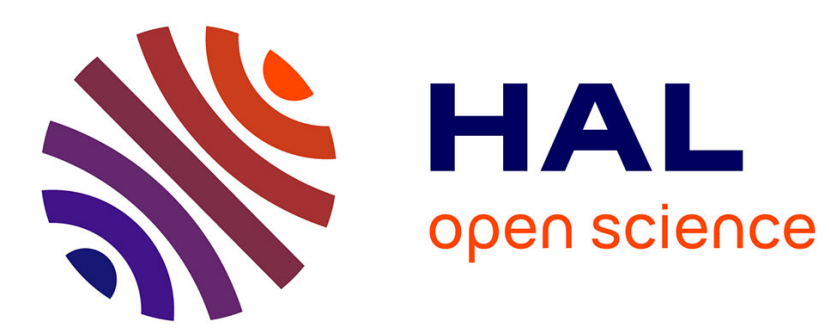

\title{
Breaking of Ergodicity in Expanding Systems of Globally Coupled Piecewise Affine Circle Maps
}

Bastien Fernandez

\section{To cite this version:}

Bastien Fernandez. Breaking of Ergodicity in Expanding Systems of Globally Coupled Piecewise Affine Circle Maps. Journal of Statistical Physics, 2014, 154 (4), pp.999-1029. 10.1007/s10955-013-0903-9 . hal-00903237

\section{HAL Id: hal-00903237 https://hal.science/hal-00903237}

Submitted on 10 Nov 2013

HAL is a multi-disciplinary open access archive for the deposit and dissemination of scientific research documents, whether they are published or not. The documents may come from teaching and research institutions in France or abroad, or from public or private research centers.
L'archive ouverte pluridisciplinaire HAL, est destinée au dépôt et à la diffusion de documents scientifiques de niveau recherche, publiés ou non, émanant des établissements d'enseignement et de recherche français ou étrangers, des laboratoires publics ou privés. 


\title{
Breaking of Ergodicity in Expanding Systems of Globally Coupled Piecewise Affine Circle Maps
}

\author{
Bastien Fernandez \\ Centre de Physique Théorique \\ CNRS - Aix-Marseille Université - Université de Toulon \\ Campus de Luminy \\ 13288 Marseille CEDEX 09 France
}

\begin{abstract}
To identify and to explain coupling-induced phase transitions in Coupled Map Lattices (CML) has been a lingering enigma for about two decades. In numerical simulations, this phenomenon has always been observed preceded by a lowering of the Lyapunov dimension, suggesting that the transition might require changes of linear stability. Yet, recent proofs of co-existence of several phases in specially designed models work in the expanding regime where all Lyapunov exponents remain positive.

In this paper, we consider a family of CML composed by piecewise expanding individual map, global interaction and finite number $N$ of sites, in the weak coupling regime where the CML is uniformly expanding. We show, mathematically for $N=3$ and numerically for $N \geqslant 3$, that a transition in the asymptotic dynamics occurs as the coupling strength increases. The transition breaks the (Milnor) attractor into several chaotic pieces of positive Lebesgue measure, with distinct empiric averages. It goes along with various symmetry breaking, quantified by means of magnetization-type characteristics.

Despite that it only addresses finite-dimensional systems, to some extend, this result reconciles the previous ones as it shows that loss of ergodicity/symmetry breaking can occur in basic CML, independently of any decay in the Lyapunov dimension.
\end{abstract}

August 28, 2013.

\section{Introduction}

Coupled Map Lattices (CML) are simple deterministic models of spatially extended systems composed of interacting units. Originally designed to facilitate numerical simulations, these models consist of transformations acting on real vectors (or sequences) and generated by the composition of the direct product of a one-dimensional map (representing the paralelised individual dynamics of each unit) and a coupling operator that mimics interactions between units [22]. Many variations on the theme can be found in the literature. The most common example is the following space-time discrete version of the basic reaction-diffusion equation on a periodic 1D medium

$$
x_{s}^{t+1}=(1-\epsilon) f\left(x_{s}^{t}\right)+\frac{\epsilon}{2}\left(f\left(x_{s-1}^{t}\right)+f\left(x_{s+1}^{t}\right)\right), \forall s \in \mathbb{Z} / N \mathbb{Z},
$$

where $f: \mathbb{R} \rightarrow \mathbb{R}$ is the individual map and where $\epsilon \in[0,1]$ is the coupling strength.

Being suitably adapted to explore modifications of dynamical behaviors upon variations of parameters (of the interaction strength in particular), CML have been employed in the modeling of a large 
variety of situations, from chemical oscillators [40] to populations dynamics [38] and atmospheric circulation [29], to cite few examples.

While CML are easy to implement numerically, the analysis of their dynamics faces several challenging issues [10]. As a result, the current rigorous (mathematical) description of their global properties is mostly limited to extreme parameter regimes, either when the individual dynamics dominates (uncoupled regime) or when the interaction prevails (synchronisation and the like). By focusing on small lattices (basically with 2 sites), few papers have nonetheless managed to cover the full coupling range $[15,25,28]$.

This paper aims to provide insights into the global dynamics of chaotic CML with arbitrary number of sites, beyond the uncoupled regime (i.e. when the system ceases to share the same features of its uncoupled limit), but before any change in linear (transverse) stability.

A basic result in the theory of dynamical systems is the existence of a semi-conjugacy between any map possessing a Markov partition and its associated topological Markov chain, see e.g. [23]. In other words, the dynamics of any suitable map $f$ can be regarded as an evolving discrete 'spin' with finitely many states. When applied to lattice dynamical systems such as CML, this correspondence suggests to represent these systems as spin lattices of statistical mechanics (with the coupling strength $\epsilon$ playing the role of the inverse of a temperature) [8].

While this connection to spin systems can be proved for any (piecewise) expanding CML [20], for standard models, the transition rules (grammar) of the resulting symbolic systems are usually intractable and of infinite range. Exceptions to this failure are repellers of weakly coupled chains of maps with Cantor repelling set $[12,13]$ and specially designed CML for which the coupling operator preserves the uncoupled Markov partition [2, 8, 14, 17, 21, 35, 36, 39]. Independently of grammatical issues, proofs of uniqueness of the physical measure in the weak coupling regime (analogue to the uniqueness of the high temperature phase) have been provided using perturbative approaches from the uncoupled limit $[1,5,6,16,19,27,28]$.

More intriguingly, the correspondence with spin systems indicates that phase transitions should take place in CML as the coupling strength increases [8]. In fact, a similar to the 2D Ising ferromagnetic transition had been numerically observed in some square lattices of coupled piecewise affine maps [31]. Subsequent studies revealed that this symmetry breaking transition occurs in a regime where the CML is no longer expanding, because it is preceded by a reduction of the Lyapunov dimension [3, 4]. This reduction, which is reminiscent of synchrony phenomena in coupled chaotic dynamical systems, including CML [20], casts doubt on the nature of the observed transition. Is this symmetry breaking the finite dimensional signature of the emergence, at the thermodynamic limit, of several probability measures supported on a topologically mixing attractor [30]? Or is it a consequence of a genuine topological change of the (finite-dimensional) CML attractor? Does it require a change of transverse stability/Lyapunov dimension as is the case for the transition to synchronisation? Can it be mathematically proved?

To the best of our knowledge, these questions have remained unanswered and the proof of existence of phase transition in standard CML is still a largely open problem. (Besides, phase transitions have been established in models with prescribed symbolic grammar mentioned above, that mimic probabilistic cellular automata for which the phenomenon had been rigorously proved $[2,14,17]$.)

In this paper, we show that coupling-induced symmetry breaking bifurcations, that exhibit characteristic phenomenological features of Ising-type transitions, occur in finite dimensional CML, at no expense of the Lyapunov or attractor dimension (and without any prerequisite on the symbolic dynamics). We bring evidence, in CML composed of $N>2$ globally coupled piecewise affine expanding circle maps, of ergodicity breaking of an absolutely continuous invariant measure (a.c.i.m.) into asymmetric Lebesgue components, as the coupling strength increases. Even though they do 
not quite address the phase transition enigma, these results indicate that the symmetry breaking induced emergence of an order parameter in chaotic CML can be of different nature than synchrony type phenomena. In particular, this phenomenon does not require any change of (linear) stability.

The paper is organised as follows. We begin with definitions and basic considerations on the dynamics in section 2. Then, we report numerical observations of symmetry breaking on temporal averages of two observables (orbits issued from random initial conditions), one for each symmetry of the family of $N$ maps $(N>2$, section 3$)$. For $N=3$, these numerics are completed by a direct visualisation in phase space. Moreover, they are confirmed by mathematical proofs of ergodicity of the a.c.i.m. at weak coupling, and of its failure when the coupling exceeds a threshold (section 4.2). The proofs rely on a thorough analysis of the Milnor attractor of a reduced mapping of the twotorus (Appendix B). For completeness, we show that ergodicity breaking fails for $N=2$. Indeed, in this case, we prove that our CML remains ergodic throughout the expanding domain (section 4.1 and Appendix A for proofs). We also describe for $N=2$ and 3, synchrony phenomena that take place in the contractive regime, and characterised by the global convergence to a 1-dimensional attractor.

Notice finally that global symmetry breaking bifurcations (a.k.a. attractor splitting crises) have already been reported in the literature $[11,18]$, mostly based on numerical simulations. Our result for $N=2$ provides a proof of such bifurcation in the case where the attractor dimension, before and past the crisis, remains equal to that of the embedding space.

\section{Globally coupled piecewise affine maps of the circle}

\subsection{Definition of the dynamics}

Given an integer $N \in \mathbb{N}$ (that represents the total number of units in the system), we consider the one-parameter family $\left\{F_{\epsilon, N}\right\}_{\epsilon \in[0,1]}$ of maps of the $N$-dimensional torus $\mathbb{T}^{N}=(\mathbb{R} / \mathbb{Z})^{N}$ into itself, defined by

$$
\left(F_{\epsilon, N}(x)\right)_{s}=2\left(x_{s}+\frac{\epsilon}{N} \sum_{r=1}^{N} g\left(x_{r}-x_{s}\right)\right)(\bmod 1), \forall s \in\{1, \cdots, N\}, x=\left(x_{s}\right)_{s=1}^{N} \in \mathbb{T}^{N},
$$

where the function $g$ mimics elastic interactions on the circle (as opposed to on the real line $\mathbb{R}$, see Figure 1 and also [28] for more details). More formally, $g$ is the function of the circle $\mathbb{T}^{1}$ that, on the domain $\left[-\frac{1}{2}, \frac{1}{2}\right]$, is given as follows ${ }^{1}$

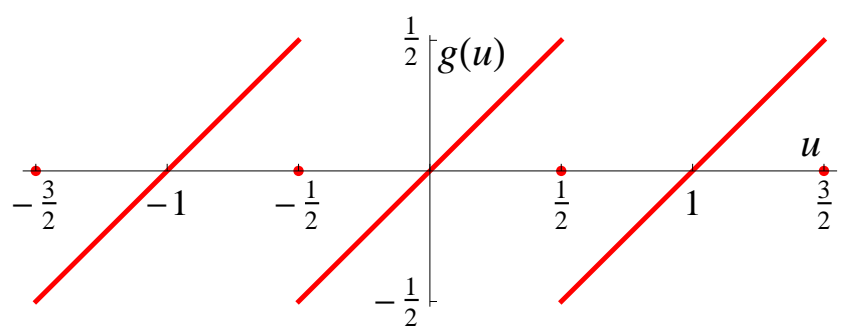

Figure 1: Graph of the function $g$ involved in the definition of the interaction term of $F_{\epsilon, N}$.

\footnotetext{
${ }^{1}$ For the sake of notation, throughout the paper, we identify real numbers with equivalence classes in the circle $\mathbb{T}^{1}$ and vice-versa, as long as no confusion results. We however keep the notation $(\bmod 1)$ in the algebraic expressions of maps in order to emphasize that we are dealing with actions of $\mathbb{T}^{N}$.
} 


$$
g(u)=\left\{\begin{array}{ccc}
u & \text { if } & |u|<\frac{1}{2} \\
0 & \text { if } & |u|=\frac{1}{2}
\end{array}, \forall u \in\left[-\frac{1}{2}, \frac{1}{2}\right]\right.
$$

Of note, $g$ has the reflection symmetry: $g(-u)=-g(u)(\bmod 1)$ for all $u \in \mathbb{T}^{1}$.

The map $F_{\epsilon, N}$ can be viewed as a crude approximation of more traditional systems where $g$ is usually assumed to be smooth, typically to be the sine function, see e.g. [20]. The current choice obviously makes the map more amenable to rigorous mathematical analysis. This applies in particular to the case $N=3$ where, to the best of the author's knowledge, no analogous results to those in section 4.2 have been published in the literature (also including coupled maps with diffusion on the real line).

The operator $C_{\epsilon, N}$ given by

$$
\left(C_{\epsilon, N}(x)\right)_{s}=x_{s}+\frac{\epsilon}{N} \sum_{r=1}^{N} g\left(x_{r}-x_{s}\right)(\bmod 1), \forall s \in\{1, \cdots, N\}, x \in \mathbb{T}^{N},
$$

which is indeed well defined as a mapping from $\mathbb{T}^{N}$ into itself - hence the same property holds for $\left.F_{\epsilon, N}\right)$ - is called the coupling operator. Furthermore, this definition of $F_{\epsilon, N}$ as the composition $F_{0, N} \circ C_{\epsilon, N}$, instead of the more standard convention $C_{\epsilon, N} \circ F_{0, N}$ adopted in the literature [10, 22], makes to our opinion the analysis easier. As the dynamics is concerned, this choice is unimportant since both dynamical systems are semi-conjugated to each other (through either $F_{0, N}$ or $C_{\epsilon, N}$ depending upon the choice of the conjugacy relation).

\subsection{Simplification of the analysis: Reduction to $1+(N-1)$ dimensions}

A simple but important feature of $F_{\epsilon, N}$ is the fact that, under a suitable change of variables, this map can be transformed into the product of two independent actions. One map is one-dimensional and has $\epsilon$-independent and well-known dynamics. The other action is $N-1$ dimensional and varies with the coupling strength parameter. To see this, let

$$
m(x)=\sum_{s=1}^{N} x_{s}, \forall x \in \mathbb{T}^{N}
$$

Then, the reflection symmetry of $g$ easily implies the following relation on $\mathbb{T}^{N}$

$$
m \circ F_{\epsilon, N}=2 m(\bmod 1),
$$

viz. the dynamics of $m$ is just the doubling of the variable on the circle, and in particular it is transitive and ergodic with respect to the Lebesgue measure (whatever value $\epsilon$ takes).

Moreover, letting $y_{s}=x_{s}-\frac{m(x)}{N}$ for all $s$, we get $\sum_{s=1}^{N} y_{s}=0$, and hence there are only $N-1$ independent variables $y_{s}$. Letting also $G_{\epsilon, N-1}$ be the map acting on the first $N-1$ variables $y_{s}$ and induced by $F_{\epsilon, N}$, i.e.

$$
\left(G_{\epsilon, N-1}(y)\right)_{s}=\left(F_{\epsilon, N}(x)\right)_{s}-\frac{m \circ F_{\epsilon, N}(x)}{N}, \forall s \in\{1, \cdots, N-1\},
$$

then one shows that its explicit expression is given by

$$
\left(G_{\epsilon, N-1}(y)\right)_{s}=2\left(y_{s}+\frac{\epsilon}{N} \sum_{r=1}^{N-1} g\left(y_{r}-y_{s}\right)-\frac{\epsilon}{N} g\left(\sum_{r=1}^{N-1} y_{r}+y_{s}\right)\right)(\bmod 1), \forall s \in\{1, \cdots, N-1\},
$$


for all $y=\left(y_{s}\right)_{s=1}^{N-1} \in \mathbb{T}^{N-1}$. In particular, $G_{\epsilon, N-1}$ is independent of the variable $m$ and hence, is a genuine map of the torus $\mathbb{T}^{N-1}$. Accordingly, the analysis in this paper boils down to the study of the dynamics of the family of maps $\left\{G_{\epsilon, N-1}\right\}_{\epsilon \in[0,1]}$ of the $N$-1-dimensional torus. ${ }^{2}$

Every map $G_{\epsilon, N-1}$ possesses two important symmetries:

- $G_{\epsilon, N-1}$ commutes with every permutation of coordinates, viz. we have $\left(y_{s}\right)_{s=1}^{N-1} \mapsto\left(y_{\pi(s)}\right)_{s=1}^{N-1}$ for every permutation $\pi$ of $\{1, \cdots, N-1\}$,

- $G_{\epsilon, N-1}$ commutes with the inversion of coordinates, viz. we have $\left(y_{s}\right)_{s=1}^{N-1} \mapsto\left(-y_{s}(\bmod 1)\right)_{s=1}^{N-1}$.

The first symmetry is a direct consequence of the global interaction structure of the coupling operator. The second one follows from the reflection symmetries of both the function $g$ and the uncoupled mapping $F_{0, N}$.

Equation (1) together with the definition of $g$ show that every map $G_{\epsilon, N-1}$ is piecewise affine with derivative (outside discontinuities) given by $\left.2(1-\epsilon) \operatorname{Id}\right|_{\mathbb{T}^{N-1}}$ (where $\left.\operatorname{Id}\right|_{\mathbb{T}^{N-1}}$ is the identity on $\mathbb{T}^{N-1}$ ). Accordingly, every map is expanding for $0 \leqslant \epsilon<\frac{1}{2}$ and contracting for $\frac{1}{2}<\epsilon \leqslant 1$. (We shall not consider the limit case when $\epsilon=\frac{1}{2}$.)

That the derivative of $G_{\epsilon, N-1}$ is a multiple of $\left.\mathrm{Id}\right|_{\mathbb{T}^{N-1}}$ implies that the so-called multiplicity entropy must vanish [9]. Focusing on the expanding domain, it follows from the ergodic theory of multidimensional dynamical systems with discontinuities that, for every $0 \leqslant \epsilon<\frac{1}{2}$, every map $G_{\epsilon, N-1}$ has an absolutely continuous (with respect to the Lebesgue measure in $\mathbb{T}^{N-1}$ ) invariant measure (a.c.i.m.) whose density is uniformly positive on some open ball $[9,37]$. Moreover there can be at most finitely many Lebesgue ergodic components, viz. ergodic components with positive Lebesgue measure.

Therefore, even though the attractor of $G_{\epsilon, N-1}$ may change as $\epsilon$ varies, it must always contain a ball in $\mathbb{T}^{N-1}$. For the original map $F_{\epsilon, N}$, it means in particular that no synchrony property (i.e. global convergence to invariant sets of dimension $<N[7,34])$ can emerge as long as $0 \leqslant \epsilon<\frac{1}{2}$. The corresponding attractor must remain 'large' (and contain open sets of $\mathbb{T}^{N}$ ) throughout the expanding domain.

Moreover, perturbation arguments on spectral gap of the transfer operator associated with standard CML $[24,26]$ and the semi-conjugacy mentioned at the end of the previous section, imply that the maps $G_{\epsilon, N-1}$ are mixing (hence ergodic) with respect to their a.c.i.m., provided that the coupling is sufficiently weak.

Accordingly, emphasis will be made here on detecting symmetry breaking bifurcations of $G_{\epsilon, N-1}$ as $\epsilon$ increases in the expanding domain. ${ }^{3}$ More precisely, we mean the emergence of several Lebesgue ergodic components, that are not invariant under the mentioned symmetries.

\section{$3 \quad$ Numerical results on symmetry breaking}

This section reports results obtained from numerical simulations of the maps $G_{\epsilon, N-1}$ across the expanding domain. These results show that, while the dynamics remains symmetric on average in a large part of the domain, multiple ergodic components with asymmetric features emerge in the long term as soon as the coupling strength exceeds some threshold. This phenomenology can be observed both for the inversion symmetry and for the symmetry of permutation of coordinates.

\footnotetext{
${ }^{2}$ More formally, the arguments here show that $F_{\epsilon, N}$ and the direct product $2 m(\bmod 1) \times G_{\epsilon, N-1}$ are in fact smoothly conjugated by the torus automorphism $x \mapsto(m, y)$.

${ }^{3}$ For $N=2$ and 3 , the dynamics in the contracting regime will also be described.
} 
In practice, orbits were generated starting from random initial conditions drawn with uniform distributions and empiric averages of asymmetry quantifiers were computed. These quantities are displayed here vs. $\epsilon$ across the expanding domain, for several values of $N$. For $N=3$, we also show direct evidence of ergodicity and then symmetry breaking, in the two-dimensional phase space of the map $G_{\epsilon, 2}$.

\subsection{Breaking the inversion symmetry}

Here, we present results related to the inversion symmetry obtained by evaluating space-time averages of coordinates (projected on the interval $\left[-\frac{1}{2}, \frac{1}{2}\right)$ ). Figure 2 reports superimposed statistical realizations of the following order parameter ${ }^{4}$

$$
\left|\frac{1}{T(N-1)} \sum_{t=0}^{T-1} \sum_{s=1}^{N-1} \pi_{\left[-\frac{1}{2}, \frac{1}{2}\right)}\left(y_{s}^{t+t_{0}}\right)\right| \text { where } y^{k}=G_{\epsilon, N-1}^{k}(y) \text { and } t_{0}=\frac{T}{5},
$$

computed for random initial conditions $y \in \mathbb{T}^{N-1}$ and three values of $T$, as the parameter $\epsilon$ runs across an interval that extends up to the boundary $\epsilon=\frac{1}{2}$ of the expanding domain.

As can be anticipated in this chaotic context, the pictures show that the average (2) indeed depends both on the initial condition and on the averaging length $T$. However, its statistical fluctuations behave as if caused by finite size effects: despite that their range gets larger when the number of initial conditions is augmented (see insets), the fluctuations decrease when the averaging length increases. Anticipating that these deviations should eventually vanish in the limit of large averaging lengths, the pictures indicate that the order parameter takes only a limited number of asymptotic values, for each value of $\epsilon$ (reminiscent of the fact that the number of Lebesgue ergodic components of $G_{\epsilon, N-1}$ is finite).

These numerics reveal the existence of a threshold $\epsilon_{c, N-1}$ for every $N$ (and $0.41<\epsilon_{c, N-1}<0.45$ ) up to which the order parameter asymptotically vanish for every initial condition. Beyond $\epsilon_{c, N-1}$, the behaviors cease to be symmetric and the order parameter converges as $T \rightarrow \infty$ to one of several nonvanishing values. Fig. 2 also suggests that each limit value is attained for a positive fraction of initial conditions (the same for the pictures and their inset), advocating that ergodicity of the physical measure [42] is likely to be broken and several asymmetric Lebesgue ergodic components emerge at $\epsilon_{c, N-1}$. Interestingly, more asymptotic values (ergodic components) appear as $\epsilon$ further increases $(N>3)$, suggesting that secondary bifurcations may take place beyond $\epsilon_{c, N-1}$. The maximum number of ergodic components definitively increases with $N$, possibly in a linear way. ${ }^{5}$ Moreover, the maximum amplitude of the order parameter decreases (by several orders of magnitude) when $N$ increases.

The same phenomenology has also been observed for other values of $N>2$, namely $N=4,6,7,40$ and 100, see Figure 3 for the latter cases (other results not shown). ${ }^{6}$ We conjecture that spontaneous symmetry breaking of the a.c.i.m./physical measure into multiple ergodic components holds for every finite number $N>2$ of sites. Beside improved algorithms, to check this conjecture for (very) large values of $N$ certainly requires powerful machines. Indeed, it took about 2 weeks CPU time

\footnotetext{
${ }^{4}$ The function $\pi_{\left[-\frac{1}{2}, \frac{1}{2}\right)}$ is the canonical projection from the circle $\mathbb{T}^{1}$ to the interval $\left[-\frac{1}{2}, \frac{1}{2}\right)$, namely $\pi_{\left[-\frac{1}{2}, \frac{1}{2}\right)}(u)=$ $\lfloor u\rfloor-\frac{1}{2}$. (No boundary effect resulting from this choice seems to affect our conclusions, as can be expected from the fact that sets of zero Lebesgue measure do not affect the dynamics of a.e. initial conditions, because all maps $G_{\epsilon, N-1}$ are non-singular, see beginning of section 4.)

${ }^{5}$ In particular, there are 5 limit values of the order parameter (2) for $N=10$, and 10 (resp. 20) values for $N=20$ (resp. 40), see also Fig. 3.

${ }^{6}$ As said before, the map $G_{\epsilon, 1}$ for $N=2$ is ergodic across the entire expanding domain (see statement 4.1 below).
} 

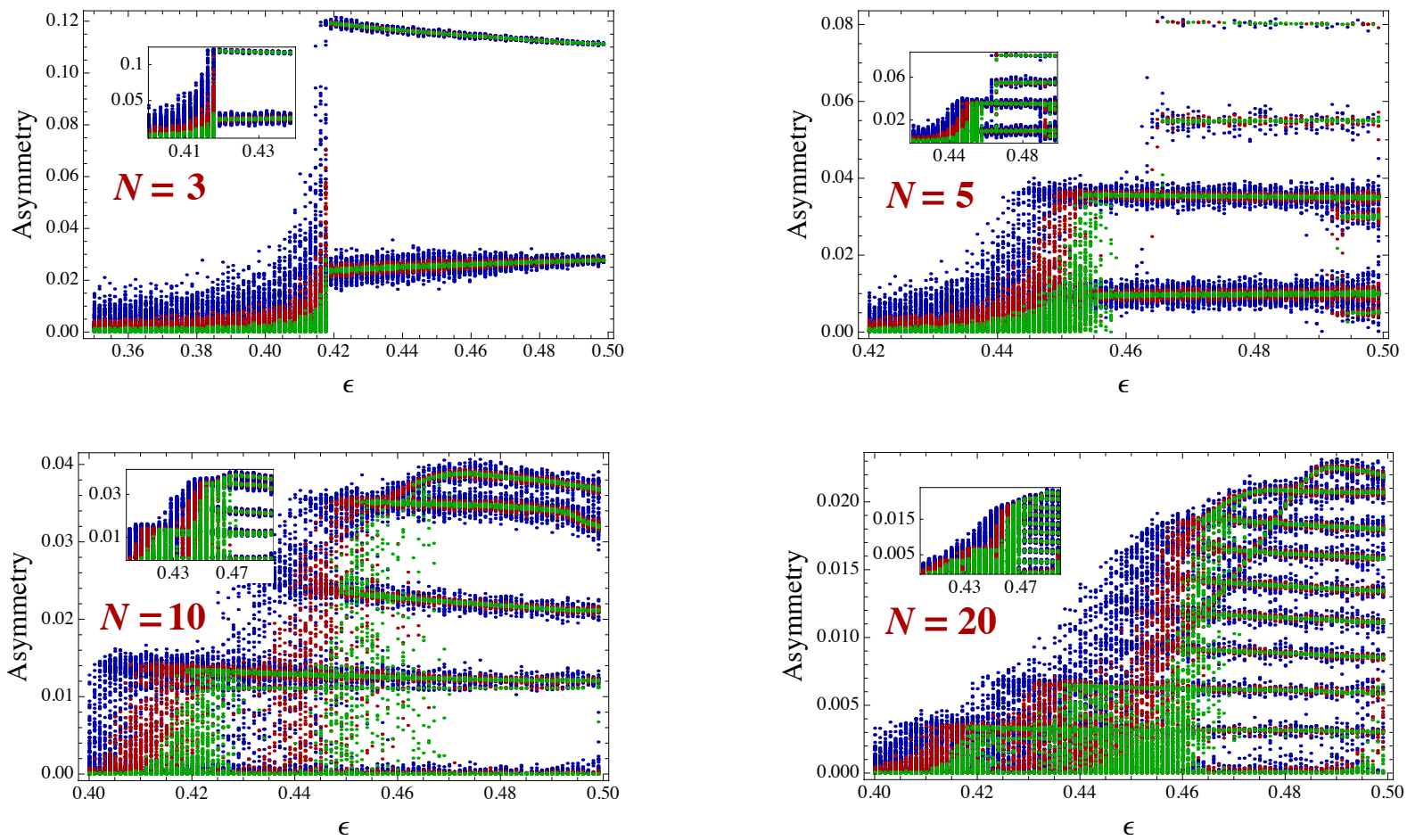

Figure 2: Superimposed statistical plots of the order parameter $\left|\frac{1}{T(N-1)} \sum_{t=0}^{T-1} \sum_{s=1}^{N-1} \pi_{\left[-\frac{1}{2}, \frac{1}{2}\right)}\left(y_{s}^{t+t_{0}}\right)\right|$ vs. $\epsilon$ in the expanding domain, for respectively $N=3,5,10$ and 20 . (The symbol $\pi_{\left[-\frac{1}{2}, \frac{1}{2}\right.}$ ) denotes the canonical projection $\mathbb{T}^{1} \rightarrow\left[-\frac{1}{2}, \frac{1}{2}\right)$ and we choose $t_{0}=\frac{T}{5}$.) Each dot corresponds to a single orbit realisation from a random initial condition drawn with uniform distribution in $\mathbb{T}^{N-1}$. Dot colors depend on the length $T$ of temporal averages (blue: $T=10^{4}$, red: $T=10^{5}$, green: $T=10^{6}$ ). For each value of $\epsilon, 100$ dots are plotted for each color; first blue, then red and finally green (insets: 500 dots are plotted for each value of $\epsilon$ ).

on QuadriCore-3.4GHz computer to obtain the results for $N=100$. In addition, the decay of the maximal amplitude and the increase of the number of ergodic components suggest that longer averaging lengths might be needed in order to reduce finite-size effects and to obtain well-defined averages and neat evidence of the existence of two regimes.

\subsection{Breaking the permutation symmetry}

Symmetry breaking in the dynamics of the maps $G_{\epsilon, N-1}$ can also be detected by means of an order parameter associated with the permutation of coordinates. To that goal, we first partition the torus $\mathbb{T}^{N-1}$ into isomorphic subsets that are mapped one into another under this symmetry, i.e. we write $^{7}$

$$
\mathbb{T}^{N-1}=\bigcup_{s=1}^{N-1} U_{s}(\bmod 0)
$$

where

$U_{s}=\left\{y \in \mathbb{T}^{N-1}: s\right.$ is the smallest $s^{\prime} \in\{0, \cdots, N-1\}$ s.t. $\left.y_{s^{\prime}}-y_{s^{\prime}+1}=\min _{r \in\{1, \cdots, N-1\}} y_{r}-y_{r+1}\right\}$.

\footnotetext{
${ }^{7}(\bmod 0)$ indicates that the symmetric difference between the corresponding sets has zero $\mathbb{T}^{N-1}$-Lebesgue measure.
} 

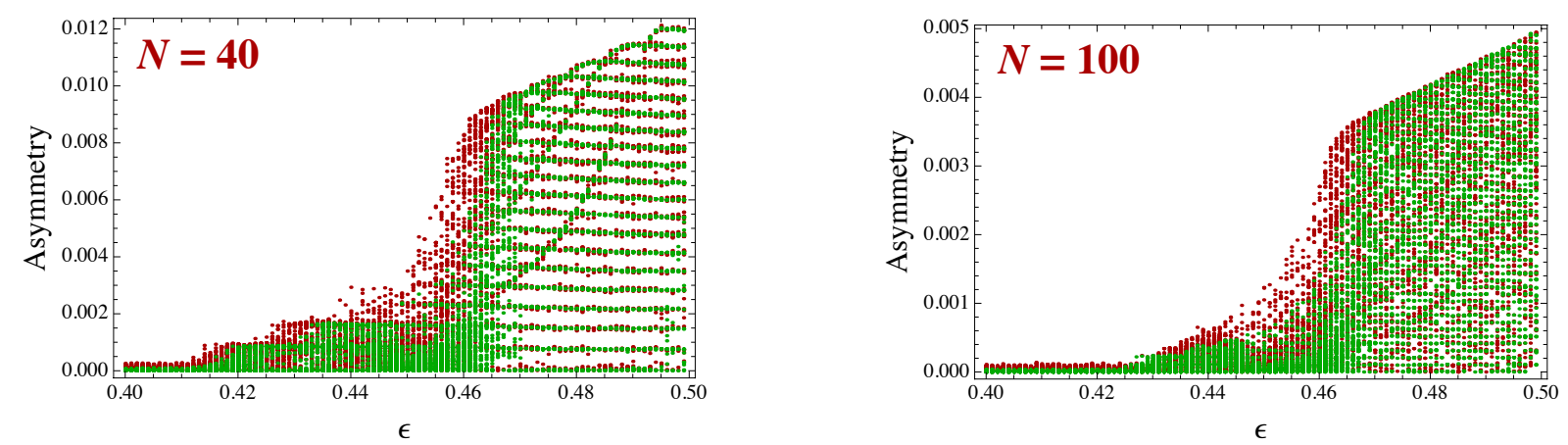

Figure 3: Superimposed statistical plots of the order parameter (2) for $N=40$ and 100. Characteristics as in Fig. 2.

Now, similarly to Fig. 2, Figure 4 reports superimposed statistical realizations of the following order parameter

$$
\left|\frac{1}{T(N-1)} \sum_{t=0}^{T-1} \sum_{s=1}^{N-1} \chi_{U_{s}}\left(y^{t+t_{0}}\right) \exp \left(\frac{2 i \pi s}{N-1}\right)\right|,
$$

where $\chi_{A}$ is the characteristic function of the set $A$. (As before, boundary effects in this definition seem to have no consequence on the statistics, thanks to $G_{\epsilon, N-1}$ being non-singular.) In short terms, this number quantifies the asymmetry of the visit frequencies into the sets $U_{s}$; it vanishes for an equidistributed orbit and it is maximal and is equal to 1 for an orbit that only visits a single $U_{s}$.

These pictures reveal similar features to the previous ones and confirm the observed phenomenology. Symmetric behavior holds on average for $\epsilon$ up to $\epsilon_{c, N-1}$ (same threshold as before), and then several Lebesgue ergodic components emerge.

Substantial differences can however be pointed out. First, Fig. 4 shows that some ergodic components beyond threshold do not break the permutation symmetry. There always exists a positive fraction of orbits for which this order parameter vanishes. Moreover, the number of asymptotic values remains small when $\epsilon$ approaches the boundary of the expanding domain - definitively smaller than the corresponding number in the previous diagnostic - and does not seem to increase with $N$.

As before, the maximum amplitude also substantially decreases when $N$ increases and appears to be comparatively smaller than before for large $N$ (compare the figures $N=10$ and 20). This maximum becomes so tiny for $N=20$ that the transition is no longer detectable with averages of length $T=10^{5}$. Lengths such as $T=10^{7}$ need to be employed in order to clearly reveal the phenomenon. In spite of this drawback, unlike the previous one, this order parameter can be employed in more general CML where the individual map/uncoupled system $F_{0, N}$ does not possess the symmetry of inversion of coordinates.

\subsection{Symmetry breaking for the map $G_{\epsilon, 2}$ attractor}

For $N=3$, the map $G_{\epsilon, 2}$ is two-dimensional. Plotting its attractor vs. $\epsilon$ provides complementary illustrative information to previous statistics. Figure 5 reports plots of the orbit components $\left\{\left(y_{1}^{t+t_{0}}, y_{2}^{t+t_{0}}\right)\right\}_{t=0}^{T-1}$ (with $T=2000$ and $t_{0}=500$ ), issued from random initial conditions in $\mathbb{T}^{2}$. The figure suggests that ergodicity holds for $\epsilon$ up to $\epsilon_{c, 2} \sim 0.417$. Indeed, every orbit densely visits a unique set with finitely many connected components. At $\epsilon=\epsilon_{c, 2}$, a bifurcation takes place 

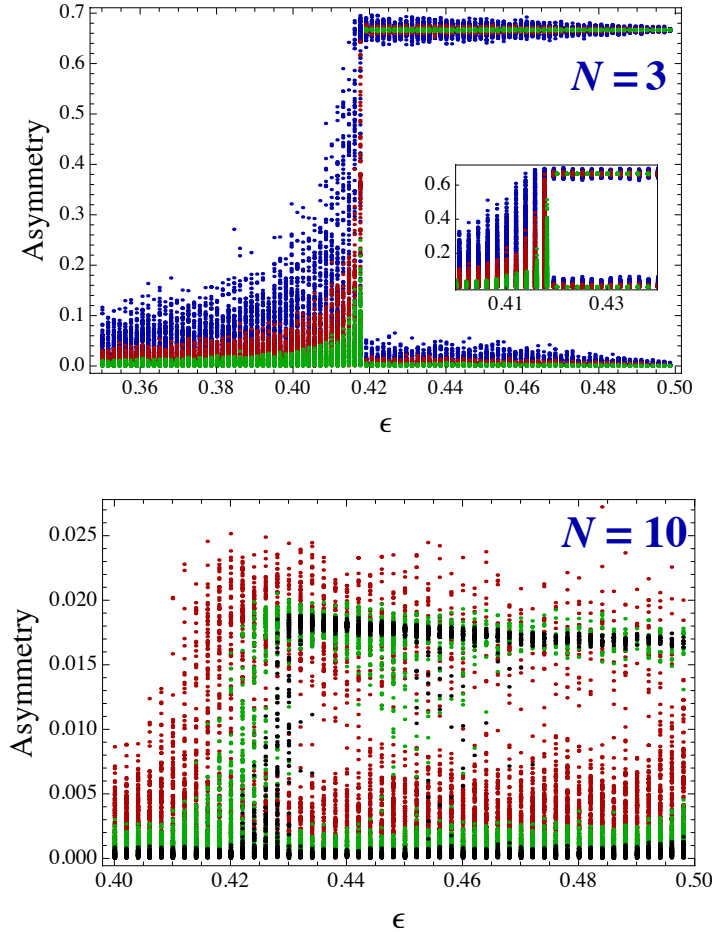

Figure 4: Superimposed statistical plots of the order parameter $\left|\frac{1}{T(N-1)} \sum_{t=0}^{T-1} \sum_{s=1}^{N-1} \chi_{U_{s}}\left(y^{t+t_{0}}\right) \exp \left(\frac{2 i \pi s}{N-1}\right)\right|$ vs. $\epsilon$ in the expanding domain (see text for definitions), for respectively $N=3,5,10$ and 20. Characteristics as in Fig. 2; black dots correspond to $T=10^{7}$.

that breaks ergodicity: 6 distinct closed invariant sets of positive Lebesgue measure emerge, that compose the attractor beyond the transition.

As already known, this phenomenon is accompanied by symmetry breaking. ${ }^{8}$ Two of these invariant sets (composed by points colored in light blue and dark blue in Fig. 5) only break the inversion symmetry (hence there can only be two such sets), confirming the findings of Fig. 4. The other four sets ('warm' colors in the pictures) break both symmetries, although the breaking of permutation symmetry is more obvious (reminiscent of the sharp difference in the order parameter amplitudes in Fig. 2). Interestingly, these numerics suggest that the number of Lebesgue ergodic components remains equal to 6 for all $\epsilon>\epsilon_{c, 2}$. Each component shrinks to a finite number of limit points as $\epsilon$ approaches $\frac{1}{2}$, and one point in each component lies on one of the discontinuities.

${ }^{8}$ The inversion symmetry of $G_{\epsilon, 2}$ materializes here as the mirror symmetry with respect to the anti-diagonal $y_{1}=-y_{2}(\bmod 1)$, whereas the permutation symmetry is represented by the mirror symmetry with respect to the diagonal $y_{1}=y_{2}(\bmod 1)$ in Figure 5. Moreover we have $\mathbb{T}^{2}=U_{1} \cup U_{2}(\bmod 0)$ where

$$
U_{1}=\left\{y \in \mathbb{T}^{2}: y_{1} \leqslant y_{2}\right\} \text { and } U_{2}=\left\{y \in \mathbb{T}^{2}: y_{2} \leqslant y_{1}\right\} .
$$



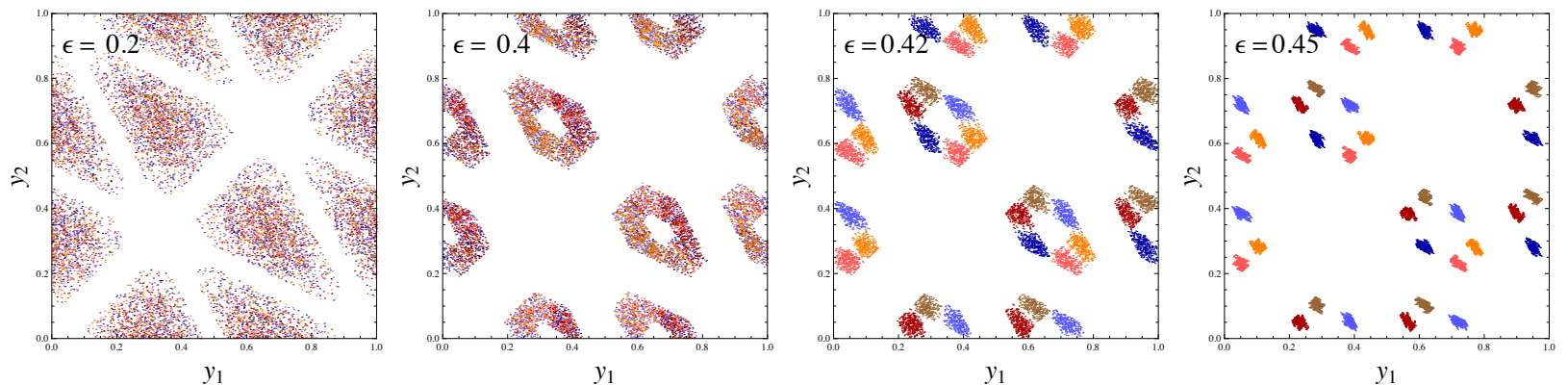

Figure 5: Plots of successive orbit components $\left\{\left(y_{1}^{t+t_{0}}, y_{2}^{t+t_{0}}\right)\right\}_{t=0}^{T-1}$ (with $T=2000$ and $t_{0}=500$ ), starting from 6 distinct initial conditions (one orbit for each color), and generated by the maps $G_{\epsilon, 2}$ for four values of $\epsilon$; two values lie below $\epsilon_{c, 2} \sim 0.417$, two lie above.

\section{Mathematical results on the dynamics of $G_{\epsilon, 1}$ and $G_{\epsilon, 2}$}

This section aims to mathematically confirm, for $N=3$, the main features of the observed phenomenology, by means of an analysis of the dynamics in the map $G_{\epsilon, 2}$ attractor. For completeness, the dynamics of $G_{\epsilon, 1}$ is also described.

Intriguingly, the symmetry breaking of $G_{\epsilon, 2}$ as observed in Fig. 5 cannot be obtained from considerations of the basic attractor

$$
\bigcap_{t=0}^{+\infty} G_{\epsilon, 2}^{t}\left(\mathbb{T}^{2}\right) .
$$

Indeed, this set consists of a single connected component for $\epsilon$ at least up to $\frac{3}{7}>\epsilon_{c, 2}$ (see beginning of section B.1 below). Moreover, even when $\epsilon$ is arbitrarily small, $G_{\epsilon, 2}$ can certainly not be transitive in this attractor because, as we shall see below, there are subsets with positive Lebesgue measure to which the dynamics never returns. (These sets are mostly repelling neighborhood of 1 -d invariant subsets.)

Since we are interested in properties of a.c.i.m., the appropriate notion of attractor in this context is due to Milnor and deals with the smallest closed set that attracts the orbit of every initial condition in $\mathbb{T}^{N-1}$, up to a set of zero Lebesgue measure [7, 32]. In particular, since every map $G_{\epsilon, N-1}$ is non-singular when $\epsilon<1,{ }^{9}$ dealing with Milnor attractor enables one to ignore those orbits that hit discontinuities, i.e. that reach, in the course of time, the set of points $y \in \mathbb{T}^{N-1}$ such that

$$
\left|y_{r}-y_{s}\right|=\frac{1}{2}(\bmod 1) \text { for some pair } r, s \in\{1, \cdots, N-1\} .
$$

More generally, all sets can be considered up to sets of zero Lebesgue measure in $\mathbb{T}^{N}$, without affecting our conclusions.

Ergodicity breaking $(N=3)$ will follow from the splitting of the Milnor attractor into asymmetric invariant sets, see statement 4.4 below and its proof in Appendix B. We begin by presenting the results for $G_{\epsilon, 1}$.

\subsection{Properties of the one-dimensional map $G_{\epsilon, 1}$}

In the case $N=2$, the map $G_{\epsilon, 1}$ is one-dimensional and writes (see Fig. 6)

$$
G_{\epsilon, 1}(y)=2 y-\epsilon g(2 y)(\bmod 1), \forall y \in \mathbb{T}^{1} .
$$

\footnotetext{
${ }^{9}$ For $\epsilon=1$, the set $G_{1, N-1}\left(\mathbb{T}^{N-1}\right)$ is finite; hence the dynamics must be eventually periodic.
} 
In Appendix A below, we show that its Milnor attractor consists of two arcs. On that set, the map is smoothly conjugated to a piecewise affine Lorenz-type map of the interval. Results on the dynamics of Lorenz maps [33, 41] immediately imply the following conclusion.

Proposition 4.1 For every $0 \leqslant \epsilon<\frac{1}{2}$, the map $G_{\epsilon, 1}$ is transitive on its Milnor attractor (that consists of two arc of the circle) and in particular, the a.c.i.m. is ergodic.

Therefore, the original CML map $F_{\epsilon, 2}$ is transitive on its Milnor attractor for every $0 \leqslant \epsilon<\frac{1}{2}$. This conclusion is the analogue of the transitivity result, obtained in [25], for a system of two doubling maps coupled by standard diffusion process on $\mathbb{R}$.

In the contracting regime $\epsilon \in\left(0, \frac{1}{2}\right)$, a straightforward analysis of the graph of $G_{\epsilon, 1}$ yields the following conclusion.

Proposition 4.2 For every $\frac{1}{2}<\epsilon<1$, we have $\lim _{t \rightarrow \infty} G_{\epsilon, 1}^{t}(y)=0$ for all $y \in \mathbb{T}^{1}$.

This result implies that global synchronization holds for the original map $F_{\epsilon, 2}$, viz. every trajectory asymptotically approaches the diagonal $x_{1}=x_{2}(\bmod 1)$. Again, this conclusion is reminiscent of the results in [25], where almost sure synchrony has been proved in the strong coupling regime. ${ }^{10}$

\subsection{Ergodic properties of the map $G_{\epsilon, 2}$}

For $N=3$, the map $G_{\epsilon, 2}$ acts on the standard torus $\mathbb{T}^{2}$ and its expression writes

$$
G_{\epsilon, 2}\left(y_{1}, y_{2}\right)=\left(2 y_{1}+\frac{2 \epsilon}{3}\left(g\left(y_{2}-y_{1}\right)-g\left(2 y_{1}+y_{2}\right)\right), 2 y_{2}+\frac{2 \epsilon}{3}\left(g\left(y_{1}-y_{2}\right)-g\left(y_{1}+2 y_{2}\right)\right)\right)(\bmod 1)
$$

for all $\left(y_{1}, y_{2}\right) \in \mathbb{T}^{2}$. Technical limitations prevent to obtain results as complete as in the onedimensional case. However, one can determine the Milnor attractor and prove its ergodicity for small coupling, and also ensure ergodicity breaking with estimate on the number of components for large coupling.

Proposition 4.3 For every $\epsilon<1-\frac{\sqrt{2}}{2} \sim 0.293$, the Milnor attractor of $G_{\epsilon, 2}$ (more precisely its intersection with the fundamental domain of $\mathbb{T}^{2}$ ) consists of a finite union of convex polygons. Moreover, $G_{\epsilon, 2}$ is transitive on this set.

Proposition 4.3 implies that the a.c.i.m. associated to the original coupled map $F_{\epsilon, 3}$ is mixing in this regime. The polygons ('islands') can be observed in the left panel of Fig. 5 and they are known explicitly, see Propositions B.10 and B.12 in Appendix B where Proposition 4.3 is proved. For convenience, the proof works with another map $H_{\epsilon, 2}$ to which $G_{\epsilon, 2}$ is conjugated by an automorphism. Moreover, the second panel in Fig. 5 indicates that the Milnor attractor is certainly not composed of convex islands sets as $\epsilon$ approaches $\epsilon_{c, 2}$; hence the restriction $\epsilon<1-\frac{\sqrt{2}}{2}$ here. In other words, any proof of ergodicity for $1-\frac{\sqrt{2}}{2} \leqslant \epsilon<\epsilon_{c, 2}$ requires additional considerations and is yet to be completed.

Although the proof fails to provide the optimal bound for ergodicity, it consists of a thorough geometrical analysis of the dynamics that paves the way to the proof of ergodicity breaking for stronger coupling. As reported above, this phenomenon materializes as the emergence of large closed asymmetric invariant sets from the previously connected islands.

\footnotetext{
${ }^{10}$ Global synchronization however does not hold for the system in [25], as a 2-periodic orbit off the diagonal exists for every $\epsilon \in[0,1][13]$.
} 
Proposition 4.4 For every $\frac{4-\sqrt{10}}{2} \sim 0.419<\epsilon<\frac{1}{2}$, every ergodic component of the map $G_{\epsilon, 2}$ must break the inversion symmetry. In particular, $G_{\epsilon, 2}$ has at least two Lebesgue ergodic components.

Moreover, for every $0.445<\epsilon<\frac{1}{2}, G_{\epsilon, 2}$ has at least six Lebesgue ergodic components.

The number $\frac{4-\sqrt{10}}{2}$ is very close to $\epsilon_{c, 2} \sim 0.417$. Moreover, the lower bound 0.445 is a three-digit upper bound approximation of the root of some cubic polynomial, see section B.2.2.

In the contracting regime $\epsilon \in\left(0, \frac{1}{2}\right)$, the dynamics is not as trivial as for $N=2$. Yet, the analysis developed in the expanding regime enables one to easily obtain the following conclusion (see section B.4).

Proposition 4.5 For every $\frac{1}{2}<\epsilon<1$, the Milnor attractor of $G_{\epsilon, 2}$ consists of the union of a stable fixed point at the origin and of three stable periodic orbits of period 2.

Of note, the periodic orbits exist for every $\epsilon \in[0,1]$ and their coordinates do not depend on the coupling strength. One periodic orbit is invariant under the inversion symmetry, one lies on the axis $y_{1}=0(\bmod 1)$ and the third one is on the on axis $y_{2}=0(\bmod 1)$. Interestingly, for $\epsilon<\frac{1}{2}$, each orbit coordinate lies at the center of one of the islands (archipelagos when $\epsilon>\epsilon_{c, 2}$ ) that appear in Fig. 5.

For the original map $F_{\epsilon, 3}$, our analysis implies that every orbit that never hits any discontinuity line, must asymptotically approach either the main diagonal $\left\{(x, x, x)(\bmod 1): x \in \mathbb{T}^{1}\right\}$ of $\mathbb{T}^{3}$ or the pair of invariant circles $\left\{x \pm\left(-\frac{1}{3}, 0, \frac{1}{3}\right)(\bmod 1): x \in \mathbb{T}^{1}\right\}$, or one of the image pair under the permutation symmetry. This result was already established in [28] (where the proof operated directly on the map $\left.F_{\epsilon, 3}\right)$.

\section{Concluding remarks}

In this paper, we have presented numerical evidences and mathematical proofs of the breaking of ergodicity of an a.c.i.m. in expanding systems of globally coupled maps. We conjecture that this phenomenon is not limited to models with mean field coupling. It should exist in any system with sufficiently long-range interactions.

That the interactions be long range appears to be necessary, at least in one-dimensional chains, because we have observed no symmetry breaking for the analogous to the maps $F_{\epsilon, N}$ with nearest neighbor coupling. Whatever the length $N>3$ of the chain is in this setting, both order parameters vanish for every coupling in the expanding domain. It would be interesting to know if the interaction range matters in higher dimensional lattices, and in particular if symmetry breaking can occur for systems of nearest neigbhor coupled maps. (As a side comment, the existence of a.c.i.m. with large support might be an issue in other models of CML, especially when the entropy of multiplicity is no longer under control throughout the expanding domain.)

Back to the globally coupled system $F_{\epsilon, N}$, an challenging question, as mentioned in section 3 , is to show numerical evidence of ergodicity breaking holds for arbitrary large $N$. Our results indicate that, when $N$ is large, longer statistics are required in order to separate the asymptotic values of the inversion symmetry order parameter. This obstacle also limits the approach based on the permutation symmetry observable. Indeed, the corresponding amplitude becomes tiny when $N$ is large, presumably because of the large (extensive) number of domains $U_{s}$ that partition the the phase space (reminiscent of absence of phase transition in models of statistical mechanics with continuous symmetry). 
As far as mathematical proofs are concerned, no proof for $N>3$ is currently available. Hence any progress in this direction would be of primary interest.

\section{Acknowledgements}

This paper would not exist if it was not for the numerous stimulating discussions I have had with L-S. Young during multiple visits at the Courant Institute (to which I am thankful for hospitality). I am also grateful to P. Ashwin (who suggested to consider the inversion symmetry), P. Collet, C. Liverani and S. Shlosman for fruitful discussions and especially to X. Leoncini for his patience and receptiveness. Financial support provided by EU Marie Curie fellowship PIOF-GA-2009-235741, CNRS PEPS Physique Théorique et ses interfaces, EU FET Projects No. MULTIPLEX 317532 and No. TOPDRIM 318121.

\section{References}

[1] V. Baladi, M. Degli Esposti, S. Isola, E. Jarvenpää, and A. Kupiainen, The spectrum of weakly coupled map lattices, J. Math. Pures App. 77 (1998), 539-584.

[2] J-B. Bardet and G. Keller, Phase transitions in a piecewise expanding coupled map lattice with linear nearest neighbour coupling, Nonlinearity 19 (2006), 2193-2210.

[3] C. Boldrighini, L. Bunimovich, G. Cosimi, S. Frigio, and A. Pellegrinotti, Ising-type transition in coupled map lattices, J. Stat. Phys. 80 (1995), 1185-1205.

[4] _ Ising-type and other transitions in one-dimensional coupled map lattices with sign symmetry, J. Stat. Phys. 102 (2001), 1271-1283.

[5] J. Bricmont and A. Kupiainen, Coupled analytic maps, Physica D 8 (1995), 379-396.

[6] _ High temperature expansions and dynamical systems, Commun. Math. Phys. 178 (1996), 703-732.

[7] J. Buescu, Exotic attractors, Birkhäuser, 1997.

[8] L. Bunimovich and Y. Sinai, Space-time chaos in coupled map lattices, Nonlinearity 1 (1988), $491-516$.

[9] J. Buzzi, Intrinsic ergodicity of affine maps in $[0,1]^{d}$, Mh. Math. 124 (1997), 97-118.

[10] J-R. Chazottes and B. Fernandez (eds.), Dynamics of coupled map lattices and of related spatially extended systems, Lect. Notes Phys., vol. 671, Springer-Verlag, 2005.

[11] P. Chossat and M. Golubitsky, Symmetry-increasing bifurcation of chaotic attractors, Physica D 32 (1988), 423-436.

[12] R. Coutinho and B. Fernandez, Extensive bounds on the entropy of repellers in expanding coupled map lattices, Ergod. Th. \& Dynam. Sys. 33 (2013), 870-895.

[13] R. Coutinho, B. Fernandez, and P. Guiraud, Symbolic dynamics of two coupled Lorenz maps: From uncoupled regime to synchronisation, Physica D 237 (2008), 2444-2462.

[14] A. de Maere, Phase transition and correlation decay in coupled map lattices, Commun. Math. Phys. 297 (2010), 229-264. 
[15] B. Fernandez and M. Jiang, Coupling two unimodal maps of simple kneading sequences, Ergod. Th. \& Dynam. Sys. 24 (2004), $107-125$.

[16] T. Fischer and H.H. Rugh, Transfer operators for coupled analytic maps, Ergod. Th. \& Dynam. Sys. 20 (2000), 109-143.

[17] G. Gielis and R. MacKay, Coupled map lattices with phase transitions, Nonlinearity 13 (2000), $867-888$.

[18] C. Grebogi, E. Ott, and J.A. Yorke, Crises, sudden changes in chaotic attractors and transient chaos, Physica D 7 (1983), 181-200.

[19] E. Jarvenpaa, A SRB-measure for globally coupled circle maps, Nonlinearity 10 (1997), 14351469.

[20] W. Just, Globally coupled maps: phase transitions and synchronization, Physica D 81 (1995), $317-340$.

[21] _ Phase transitions in coupled map lattices and in associated probabilistic cellular automata, Phys. Rev. E 74 (2006), 046209.

[22] K. Kaneko (ed.), Theory and applications of coupled map lattices, Wiley, 1993.

[23] A. Katok and B. Hasselblatt, Introduction to the modern theory of dynamical systems, Cambridge University Press, 1995.

[24] G. Keller and M. Künzle, Transfer operators for coupled map lattices, Ergod. Th. \& Dynam. Sys. 12 (1992), 297-318.

[25] G. Keller, M. Künzle, and T. Nowicki, Some phase transitions in coupled map lattices, Physica D 59 (1992), 39 - 51.

[26] G. Keller and C. Liverani, A spectral gap for coupled map lattices, Dynamics of Coupled Map Lattices and of Related Spatially Extended Systems, Lect. Notes Phys., vol. 671, SpringerVerlag, 2005, pp. 115-151.

[27]__ Uniqueness of the SRB measure for piecewise expanding weakly coupled map lattices in any dimension, Commun. Math. Phys. 262 (2006), 33 - 50.

[28] J. Koiller and L-S. Young, Coupled map networks, Nonlinearity 23 (2010), 1121-1141.

[29] P. Lind, J. Corte-Real, and J. Gallas, Modeling velocity in gradient flows with coupled-map lattices with advection, Phys. Rev. E 66 (2002), 016219.

[30] R. MacKay, Indecomposable coupled map lattices with non-unique phases, Dynamics of Coupled Map Lattices and of Related Spatially Extended Systems, Lect. Notes Phys., vol. 671, SpringerVerlag, 2005, pp. 65-94.

[31] J. Miller and D.A. Huse, Macroscopic equilibrium from microscopic irreversibility in a chaotic coupled-map lattice, Phys. Rev. E 48 (1993), 2528-2535.

[32] J. Milnor, On the concept of attractor, Commun. Math. Phys. 99 (1985), 177-195.

[33] W. Parry, The Lorenz attractor and a related population model, Ergodic Theory (M. Denker and K. Jacobs, eds.), Lec. Notes Math., vol. 729, 1979, pp. 169-187. 
[34] A. Pikovsky, M. Rosenblum, and J. Kurths, Synchronization: A universal concept in nonlinear sciences, Cambridge University Press, 2001.

[35] H. Sakaguchi, Phase transitions in coupled Bernoulli maps, Progr. Theor. Phys. 80 (1988), $7-12$.

[36] F. Sastre and G. Perez, Phase transitions in lattices of coupled chaotic maps and their dependence on the local Lyapunov exponent, Phys. Rev. E 57 (1998), 5213-5216.

[37] B. Saussol, Absolutely continuous invariant measures for multidimensional expanding maps, Israel J. Math. 116 (2000), 223-248.

[38] R. Solé, J. Valls, and J. Bascompte, Spiral waves, chaos and multiple attractors in lattice models of interacting populations, Phys. Lett. A 166 (1992), 123 - 128.

[39] K. Takeuchi and M. Sano, Role of unstable periodic orbits in phase transitions of coupled map lattices, Phys. Rev. E 75 (2007), 036201.

[40] I. Waller and R. Kapral, Spatial and temporal structure in systems of coupled nonlinear oscillators, Phys. Rev. A 30 (1984), 2047 - 2055.

[41] R. Williams, The structure of Lorenz attractors, Publ. Math. IHES 50 (1979), 73-99.

[42] L-S. Young, What are SRB measures, and which dynamical systems have them?, J. Stat. Phys. 108 (2002), 733-754.

\section{A Analysis of the dynamics of $G_{\epsilon, 1}$}

As indicated at the beginning of section 4.1, the map $G_{\epsilon, 1}: \mathbb{T}^{1} \rightarrow \mathbb{T}^{1}$ is given by $y \mapsto 2 y-$ $\epsilon g(2 y)(\bmod 1)$. An example of its graph for $\epsilon<\frac{1}{2}$ is displayed in Figure 6.

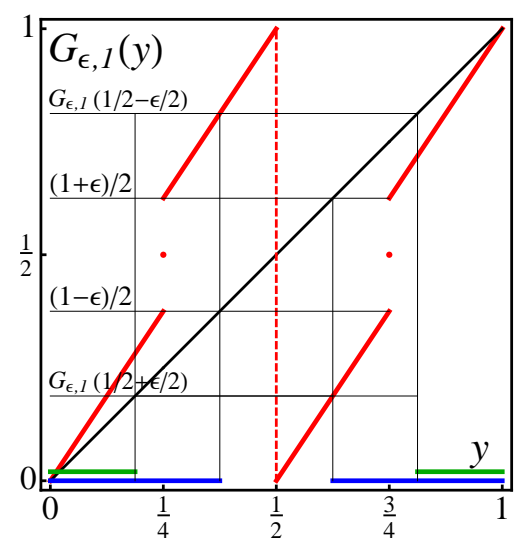

Figure 6: Graph of the map $G_{\epsilon, 1}$ of the circle in the expanding regime $\left(\epsilon=\frac{1}{4}\right)$. The horizontal blue lines represent the set $\mathcal{A}_{\epsilon}$ and the green lines materialize the set $\mathcal{B}_{\epsilon}=G_{\epsilon, 1}\left(\mathbb{T}^{1} \backslash \mathcal{A}_{\epsilon}\right)$.

In order to evaluate the action of $G_{\epsilon, 1}$, we decompose the circle into two arcs where this map is continuous, namely we write

$$
\mathbb{T}^{1}=\mathcal{I} \cup\left(\mathcal{I}+\frac{1}{2}\right)(\bmod 0),
$$

where

$$
\mathcal{I}=\left\{y \in \mathbb{T}^{1}: 0<y<\frac{1}{4}\right\} \cup\left\{y \in \mathbb{T}^{1}: \frac{3}{4}<y<1\right\}
$$


is one arc, and the other one is

$$
\mathcal{I}+\frac{1}{2}=\left\{y+\frac{1}{2}(\bmod 1): y \in \mathcal{I}\right\}=\left\{y \in \mathbb{T}^{1}: \frac{1}{4}<y<\frac{3}{4}\right\} .
$$

Now, the definition of $g$ implies $g(2 y)=2 y(\bmod 1)$ on $\mathcal{I}$; hence the set $\mathcal{A}_{\epsilon}=G_{\epsilon, 1}(\mathcal{I})$ is given by (see Fig. 6)

$$
\mathcal{A}_{\epsilon}=G_{\epsilon, 1}(\mathcal{I})=\left\{y \in \mathbb{T}^{1}: 0<y<\frac{1-\epsilon}{2}\right\} \cup\left\{y \in \mathbb{T}^{1}: \frac{1+\epsilon}{2}<y<1\right\} .
$$

Moreover, we have $G_{\epsilon, 1}\left(y+\frac{1}{2}\right)=G_{\epsilon, 1}(y)$ for all $y \in \mathbb{T}^{1}$ and together with (3) this implies

$$
G_{\epsilon, 1}\left(\mathbb{T}^{1}\right)=\mathcal{A}_{\epsilon}(\bmod 0) .
$$

A simple calculation shows that the inclusion $\mathcal{I} \subset \mathcal{A}_{\epsilon}$ holds for every $\epsilon$ in the expanding domain. Together with the previous arguments, this implies

$$
G_{\epsilon, 1}\left(\mathcal{A}_{\epsilon}\right)=\mathcal{A}_{\epsilon}(\bmod 0),
$$

i.e. $\mathcal{A}_{\epsilon}$ is the largest invariant set of the map $G_{\epsilon, 1}$. However, as announced before, this set is not the minimal attractor in the Milnor sense because it contains a subset $\mathcal{B}_{\epsilon}$ to which no orbit can ever return. This subset is defined by (see Fig. 6 again)

$$
\mathcal{B}_{\epsilon}=G_{\epsilon, 1}\left(\mathbb{T}^{1} \backslash \mathcal{A}_{\epsilon}\right)=\left\{y \in \mathbb{T}^{1}: 0<y \leqslant G_{\epsilon, 1}\left(\frac{1+\epsilon}{2}\right)\right\} \cup\left\{y \in \mathbb{T}^{1}: G_{\epsilon, 1}\left(\frac{1-\epsilon}{2}\right) \leqslant y<1\right\} .
$$

One checks that

$$
G_{\epsilon, 1}^{-1}\left(\mathcal{B}_{\epsilon}\right) \subset \mathcal{B}_{\epsilon} \cup\left(\mathbb{T}^{1} \backslash \mathcal{A}_{\epsilon}\right),
$$

viz. the only way a point $y \in \mathcal{A}_{\epsilon}$ be mapped into $\mathcal{B}_{\epsilon}$ is that the point $y$ be already in this set. Since the only point whose orbit always remains in $\mathcal{B}_{\epsilon}$ is the origin $0(\bmod 1)$, it results that the Milnor attractor of $G_{\epsilon, 1}$ must be contained in the set

$$
\mathcal{A}_{\epsilon} \backslash \mathcal{B}_{\epsilon}=\left\{y \in \mathbb{T}^{1}: G_{\epsilon, 1}\left(\frac{1+\epsilon}{2}\right)<y<\frac{1-\epsilon}{2}\right\} \cup\left\{y \in \mathbb{T}^{1}: \frac{1+\epsilon}{2}<y<G_{\epsilon, 1}\left(\frac{1-\epsilon}{2}\right)\right\} .
$$

Clearly, the set $\mathcal{A}_{\epsilon} \backslash \mathcal{B}_{\epsilon}$ consists of two disjoint arcs and

$$
G_{\epsilon, 1}\left(\mathcal{A}_{\epsilon} \backslash\left(\mathcal{B}_{\epsilon} \cup\left\{\frac{1}{4}, \frac{3}{4}\right\}\right)\right)=\mathcal{A}_{\epsilon} \backslash \mathcal{B}_{\epsilon}(\bmod 0) .
$$

By applying a change of variable that consists in gluing together the inner boundaries of the two arcs, the positive jump discontinuities of the map at $y=\frac{1}{4}$ and $y=\frac{3}{4}$ can be removed, and the resulting map turns out to be a symmetric piecewise affine Lorenz map.

More precisely, let $I_{\epsilon}$ be the open interval in $\mathbb{R}$ defined by

$$
I_{\epsilon}=\left(G_{\epsilon, 1}\left(\frac{1+\epsilon}{2}\right), G_{\epsilon, 1}\left(\frac{1-\epsilon}{2}\right)-\epsilon\right),
$$

and let the Lorenz map $H_{\epsilon, 1}: I_{\epsilon} \backslash\left\{\frac{1-\epsilon}{2}\right\} \rightarrow I_{\epsilon}$ be defined by (Figure 7)

$$
H_{\epsilon, 1}(u)=\left\{\begin{array}{cl}
2(1-\epsilon) u & \text { if } u<\frac{1-\epsilon}{2} \\
2(1-\epsilon)\left(u-\frac{1-\epsilon}{2}\right)+\epsilon(1-\epsilon) & \text { if } \quad \frac{1-\epsilon}{2}<u
\end{array}\right.
$$

Let also $r_{\epsilon}: \mathcal{A}_{\epsilon} \backslash \mathcal{B}_{\epsilon} \rightarrow I_{\epsilon} \backslash\left\{\frac{1-\epsilon}{2}\right\}$ be the change of variable given by

$$
r_{\epsilon}(u)=\left\{\begin{array}{clc}
u & \text { if } & u<\frac{1-\epsilon}{2} \\
u-\epsilon & \text { if } & \frac{1-\epsilon}{2}<u
\end{array}\right.
$$

The transformation $r_{\epsilon}$ is a diffeomorphism from $\mathcal{A}_{\epsilon} \backslash \mathcal{B}_{\epsilon}$ to $I_{\epsilon} \backslash\left\{\frac{1-\epsilon}{2}\right\}$, and the conjugacy between $G_{\epsilon, 1}$ on $\mathcal{A}_{\epsilon} \backslash \mathcal{B}_{\epsilon}$ and $H_{\epsilon}$ on $I_{\epsilon}$ (discontinuities discarded) is given by the following formal statement

Claim A.1 We have $H_{\epsilon, 1} \circ r_{\epsilon}=r_{\epsilon} \circ G_{\epsilon, 1}$ for all $\epsilon \in\left(0, \frac{1}{2}\right)$.

The proof is a simple calculation and is left to the reader.

The properties of the dynamics of $G_{\epsilon, 1}$, in particular transitivity and ergodicity everywhere in the expanding domain (Proposition 4.1 in section 4.1), are then immediate consequences of the analogous results for symmetric piecewise affine Lorenz maps, see [33] for a detailed description. 


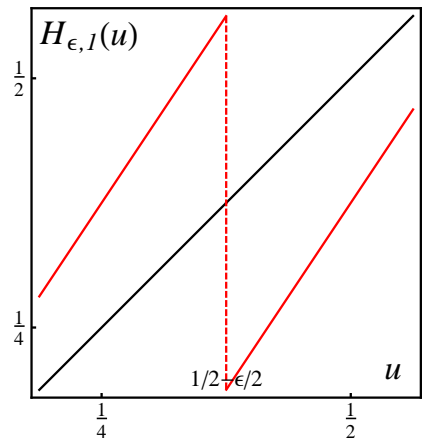

Figure 7: Graph of the Lorenz map $H_{\epsilon, 1}$ defined on the real set $I_{\epsilon} \backslash\left\{\frac{1-\epsilon}{2}\right\}\left(\epsilon=\frac{1}{4}\right)$.

\section{B Proofs of recurrent properties of the map $G_{\epsilon, 2}$}

This appendix is devoted to the proofs of Proposition 4.3 and 4.4 in section 4.2. The map $G_{\epsilon, 2}$ is piecewise affine of $\mathbb{T}^{2}$ with nine atoms on the fundamental domain. For the sake of simplicity, we first reduce the number of atoms to consider by applying the linear automorphism $M=\left(\begin{array}{cc}\frac{3}{2} & 0 \\ \frac{1}{2} & 1\end{array}\right)$. Letting $\left(\begin{array}{c}u \\ v\end{array}\right)=M\left(\begin{array}{l}y_{1} \\ y_{2}\end{array}\right)$, the induced map $H_{\epsilon, 2}=M \circ G_{\epsilon, 2} \circ M^{-1}$ of the unit torus $\mathbb{T}^{2}$ into itself writes

$$
H_{\epsilon, 2}(u, v)=\left(2 u-\epsilon(g(u+v)+g(u-v)), 2 v-\frac{\epsilon}{3}(g(u+v)+g(v-u)+2 g(2 v))\right)(\bmod 1) .
$$

As for $G_{\epsilon, 2}$, the map $H_{\epsilon, 2}$ is piecewise affine with derivative (off discontinuities) given by $\left.2(1-\epsilon) \operatorname{Id}\right|_{\mathbb{T}^{2}}$. Hence, this map also has an a.c.i.m. with density uniformly positive on some open ball, for every $\epsilon$ in the expanding domain. From now on, we exclusively consider $H_{\epsilon, 2}$ instead of $G_{\epsilon, 2}$.

\section{B.1 Milnor attractor of the map $H_{\epsilon, 2}$}

This section contains the construction of the Milnor attractor of $H_{\epsilon, 2}$. More exactly, we construct the smallest systematically attracting and recurrent set of this map, see Proposition B.10 as the end of this section. As $G_{\epsilon, 1}\left(\mathbb{T}^{1}\right)$ was invariant under $G_{\epsilon, 1},{ }^{11}$ the image $H_{\epsilon, 2}\left(\mathbb{T}^{2}\right)$ of the whole phase space turns out to be invariant (at least when $\epsilon<\frac{3}{7}$ ). However, this set has several non-recurrent subregions. Accordingly, the construction consists in identifying and in removing these subregions, one after another, until no further reduction is feasible.

The map $H_{\epsilon, 2}$ commutes with the reflexion symmetries $S_{u}$ and $S_{v}$ respectively defined by

$$
S_{u}(u, v)=(-u, v)(\bmod 1) \text { and } S_{v}(u, v)=(u,-v)(\bmod 1), \forall(u, v) \in \mathbb{T}^{2} .
$$

(The composition $S_{u} \circ S_{v}$ is nothing but the inversion of both coordinates inherited from $G_{\epsilon, 2}$.) Given a subset $X \subset \mathbb{T}^{2}$, we shall denote by $O(X)=X \cup S_{u}(X) \cup S_{v}(X) \cup S_{u} \circ S_{v}(X)$, the orbit of this set under the associated group. Throughout the construction, we shall also frequently take advantage of the following translation invariance

$$
H_{\epsilon, 2}\left(u+\frac{1}{2}, v+\frac{1}{2}\right)=H_{\epsilon, 2}(u, v), \forall(u, v) \in \mathbb{T}^{2} .
$$

\footnotetext{
${ }^{11}$ Several properties of $H_{\epsilon, 2}$ are actually two-dimensional analogue of properties of the map $G_{\epsilon, 1}$.
} 
The torus $\mathbb{T}^{2}$ decomposes into six domains where $H_{\epsilon, 2}$ is continuous (Figure 8). These atoms are generated by two basic polygons; one is the following trapezoid

$$
\mathrm{I}=\left\{(u, v) \in \mathbb{T}^{2}: 0<u<\frac{1}{2}, 0<v<\frac{1}{4}, u+v<\frac{1}{2}\right\}
$$

and the other one is the triangle

$$
\mathrm{II}=\left\{(u, v) \in \mathbb{T}^{2}: 0<u<\frac{1}{4}, \frac{1}{4}<v<\frac{1}{2}, u+v<\frac{1}{2}\right\} .
$$

Now, the decomposition writes

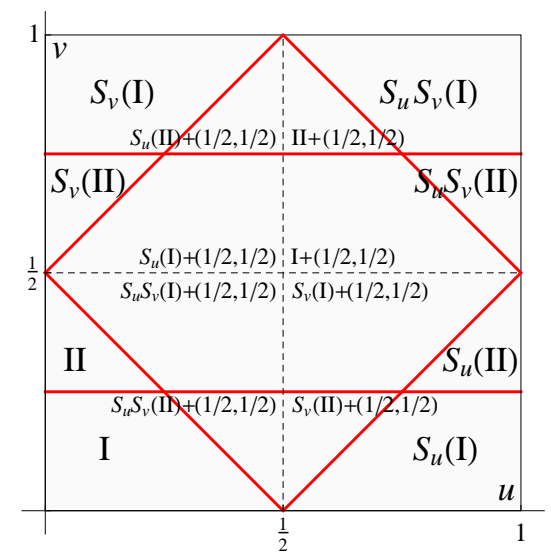

Figure 8: Decomposition of the torus $\mathbb{T}^{2}$ into continuity atoms for $H_{\epsilon, 2}$. One atom is $\mathcal{I}_{1}=O(\mathrm{I})=$ $\mathrm{I} \cup S_{u}(\mathrm{I}) \cup S_{v}(\mathrm{I}) \cup S_{u} \circ S_{v}(\mathrm{I})$; one atom is $\mathcal{I}_{2}=\mathrm{II} \cup S_{u}(\mathrm{II})$; one atom is the symmetric image $\mathcal{I}_{3}=S_{v}\left(\mathcal{I}_{2}\right)$ and the three other atoms are obtained by applying the translation $X \mapsto X+\left(\frac{1}{2}, \frac{1}{2}\right)=$ $\left\{\left(u+\frac{1}{2}, v+\frac{1}{2}\right)=(u, v) \in X\right\}$. The solid (red) segments represent discontinuity lines, respectively $\mathbb{T}^{1} \times\left\{\frac{1}{4}\right\}$ and $\mathbb{T}^{1} \times\left\{\frac{3}{4}\right\}$ for the horizontal lines and $D=\left\{(u, v) \in \mathbb{T}^{2}: u \pm v=\frac{1}{2}(\bmod 1)\right\}$ for the four inclined lines.

$$
\mathbb{T}^{2}=\mathcal{I}_{1} \cup \mathcal{I}_{2} \cup \mathcal{I}_{3} \cup\left(\left(\mathcal{I}_{1} \cup \mathcal{I}_{2} \cup \mathcal{I}_{3}\right)+\left(\frac{1}{2}, \frac{1}{2}\right)\right)(\bmod 0)
$$

where

$$
\mathcal{I}_{1}=O(\mathrm{I}), \mathcal{I}_{2}=\mathrm{II} \cup S_{u}(\mathrm{II}) \text { and } \mathcal{I}_{3}=S_{v}\left(\mathcal{I}_{2}\right),
$$

and $H_{\epsilon, 2}$ is continuous on each $\mathcal{I}_{i}$ and each $\mathcal{I}_{i}+\left(\frac{1}{2}, \frac{1}{2}\right)$.

Borrowing a notation from the previous section, consider the set

$$
\mathcal{A}_{\epsilon}=H_{\epsilon, 2}\left(\mathcal{I}_{1} \cup \mathcal{I}_{2} \cup \mathcal{I}_{3}\right) .
$$

The decomposition (4) implies we have $\mathcal{A}_{\epsilon}=H_{\epsilon, 2}\left(\mathbb{T}^{2}\right)(\bmod 0)$; hence the attractor is certainly contained in this set. From the expression of $H_{\epsilon, 2}$ above, we get

$$
H_{\epsilon, 2}(u, v)=2(1-\epsilon)(u, v), \forall(u, v) \in \mathrm{I},
$$

and

$$
H_{\epsilon, 2}(u, v)=2(1-\epsilon)(u, v)+\left(0, \frac{2 \epsilon}{3}\right), \forall(u, v) \in \text { II. }
$$

Using these expressions and the symmetries, one obtains the following relation (Figure 9)

$$
\mathcal{A}_{\epsilon}=\mathbb{T}^{2} \backslash\left(\mathcal{S}_{\epsilon} \cup O\left(\mathcal{P}_{\epsilon}\right)\right)
$$



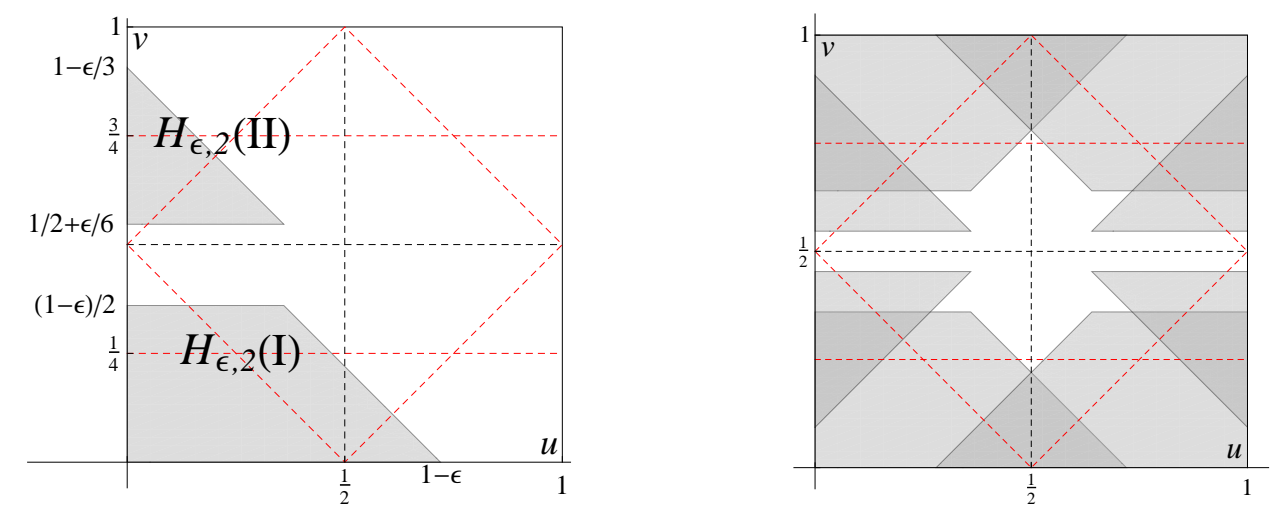

Figure 9: Images $H_{\epsilon, 2}(\mathrm{I})$ and $H_{\epsilon, 2}(\mathrm{II})$ of the basic sets (left panel) and image $\mathcal{A}_{\epsilon}=H_{\epsilon, 2}\left(\mathbb{T}^{2}\right)(\bmod 0)$ of the full torus (right panel) for $\epsilon=0.28$. The latter follows from applying symmetries to the former. No point can be mapped in the white region of the right panel (union of the strip $\mathcal{S}_{\epsilon}$ and of the four concave pentagons $O\left(\mathcal{P}_{\epsilon}\right)$ ). This picture also indicates the regions where points have respectively two pre-images (light grey) and four pre-images (dark grey). Discontinuity lines are repeated for reference.

where $\mathcal{S}_{\epsilon}$ is a strip around the axis $\mathbb{T}^{1} \times\left\{\frac{1}{2}\right\}$, i.e.

$$
\mathcal{S}_{\epsilon}=\left\{(u, v) \in \mathbb{T}^{2}: \frac{1}{2}-\frac{\epsilon}{6}<v<\frac{1}{2}+\frac{\epsilon}{6}\right\}
$$

and $\mathcal{P}_{\epsilon}$ is a concave pentagon adjacent to this strip.

As announced above, even though $\mathcal{A}_{\epsilon}$ is invariant (at least when $\epsilon<\frac{3}{7}$ ), ${ }^{12}$ this set cannot be the Milnor attractor of $H_{\epsilon, 2}$. Indeed, consider the following horizontal strip around the axis $\mathbb{T}^{1} \times\{0(\bmod 1)\}$

$$
\mathcal{B}_{\epsilon}=\left\{(u, v) \in \mathbb{T}^{2}: 0 \leqslant v<(1-\epsilon) \frac{\epsilon}{3}\right\} \cup\left\{(u, v) \in \mathbb{T}^{2}: 1-(1-\epsilon) \frac{\epsilon}{3}<v \leqslant 1\right\} .
$$

Claim B.1 We have $H_{\epsilon, 2}^{-1}\left(\mathcal{B}_{\epsilon}\right) \subset \mathcal{B}_{\epsilon} \cup\left(\mathbb{T}^{2} \backslash \mathcal{A}_{\epsilon}\right)$ for all $\epsilon<\frac{1}{2}$.

Proof: The set $\mathcal{B}_{\epsilon}$ is defined as the largest horizontal strip that contains the image of the restriction of $\mathcal{S}_{\epsilon}$ to the central atom of the partition, i.e. $H_{\epsilon, 2}\left(\mathcal{S}_{\epsilon} \cap\left(\mathcal{I}+\left(\frac{1}{2}, \frac{1}{2}\right)\right)\right)$. By translation invariance, this image coincides with $H_{\epsilon, 2}\left(\left(\mathcal{S}_{\epsilon}+\left(\frac{1}{2}, \frac{1}{2}\right)\right) \cap \mathcal{I}\right)$.

The strip $\mathcal{S}_{\epsilon}+\left(\frac{1}{2}, \frac{1}{2}\right)$ is actually contained in $\mathcal{B}_{\epsilon}$. This shows that $\mathcal{B}_{\epsilon}$ has pre-images that are located either inside $\mathcal{B}_{\epsilon}$ or in $\mathcal{S}_{\epsilon}$. Moreover, we have $\mathcal{S}_{\epsilon} \subset \mathbb{T}^{2} \backslash \mathcal{A}_{\epsilon}$. In order to conclude the proof, it suffices to show that there are no pre-images elsewhere. This is granted by the trivial inequality $(1-\epsilon) \frac{\epsilon}{3}<\frac{\epsilon}{3}$ which implies $\mathcal{B}_{\epsilon}$ does not intersect the images of the triangular sets $\mathcal{I}_{2}$ and $\mathcal{I}_{3}$. In other words, $\mathcal{B}_{\epsilon}$ can only intersect the images of $\mathcal{I}_{1}$ and $\mathcal{I}_{1}+\left(\frac{1}{2}, \frac{1}{2}\right)$ and we are done.

Claim B.1 implies no orbit can return to $\mathcal{B}_{\epsilon}$ after it has exited this set. Since the only orbits staying forever in this set are the one included in the horizontal axis $v=0(\bmod 1)$; a set that has zero Lebesgue measure in $\mathbb{T}^{2}$, viz. the Milnor attractor must be included in $\mathcal{A}_{\epsilon} \backslash \mathcal{B}_{\epsilon}$, see Figure 10.

\footnotetext{
${ }^{12}$ As long as $H_{\epsilon, 2}$ is expanding, the image $H_{\epsilon, 2}\left(\mathcal{I}_{1}\right)$ covers $\mathcal{I}_{1}$. The decomposition (4) then implies a sufficient condition for invariance of $\mathcal{A}_{\epsilon}$ is that, for every $(u, v) \in \mathrm{II}$, we have

$$
\text { either }(u, v) \in \mathcal{A}_{\epsilon} \text { or }(u, v)+\left(\frac{1}{2}, \frac{1}{2}\right) \in \mathcal{A}_{\epsilon} \text { (or both). }
$$

Direct calculations show that this property holds when $\epsilon<\frac{3}{7}$.
} 

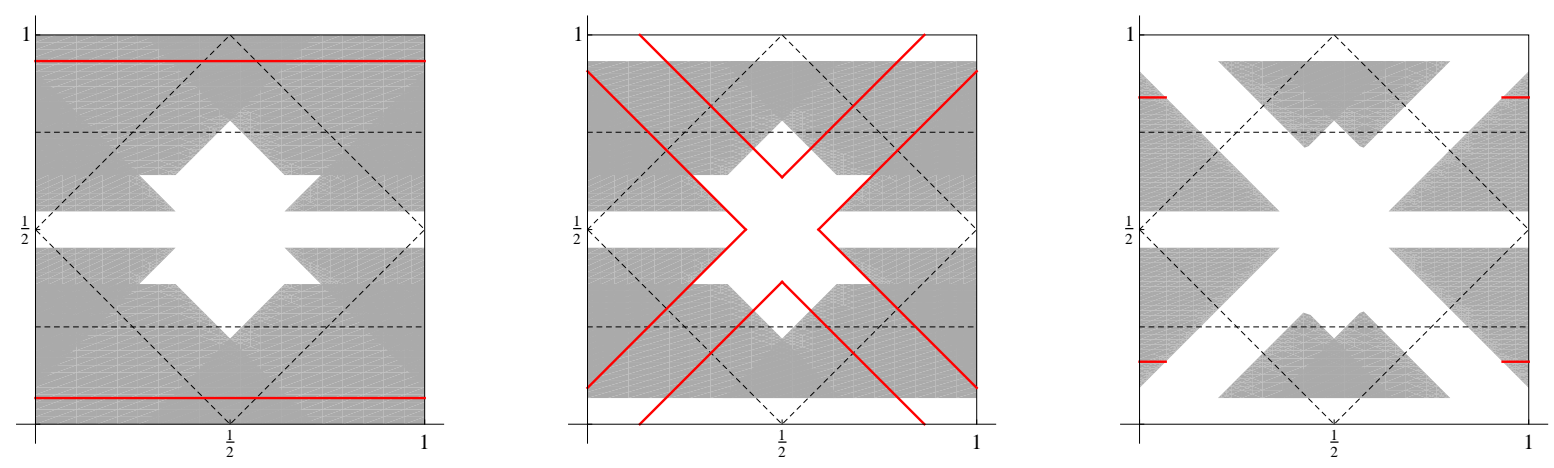

Figure 10: Construction of the Milnor attractor by successive elimination of non-recurrent regions. Left panel. Original set $\mathcal{A}_{\epsilon}=H_{\epsilon, 2}\left(\mathbb{T}^{2}\right)=\mathbb{T}^{2} \backslash\left(\mathcal{S}_{\epsilon} \cup O\left(\mathcal{P}_{\epsilon}\right)\right)$ (grey region) and boundaries (red lines) of the strip $\mathcal{B}_{\epsilon}$. Center panel. The set $\mathcal{A}_{\epsilon} \backslash \mathcal{B}_{\epsilon}$ (grey region) and boundaries (red lines) of the strips $\mathcal{C}_{\epsilon}$. Right panel. The set $\mathcal{A}_{\epsilon} \backslash\left(\mathcal{B}_{\epsilon} \cup \mathcal{C}_{\epsilon}\right)$ (grey region) and boundaries (red segments) of the four triangles $\mathcal{D}_{\epsilon}$.

The strip $\mathcal{B}_{\epsilon}$ has been obtained as the neighborhood of a (repelling) one-dimensional invariant set that can only be reached from the region $\mathcal{I}_{1} \cup \mathcal{I}_{1}+\left(\frac{1}{2}, \frac{1}{2}\right)$ of phase space. The same criterion can be applied to other sets, in particular to the intersection of the images $H_{\epsilon, 2}\left(O\left(\mathcal{P}_{\epsilon}\right)\right)$ of the pentagons above with the same region. Nonetheless, one can obtain a larger set ${ }^{13}$ by considering an appropriate neighborhood of the main diagonal $\left\{(u, u): u \in \mathbb{T}^{1}\right\}$. Let $\mathcal{C}_{\epsilon}$ be the following set (Fig. 10)

$$
\mathcal{C}_{\epsilon}=O\left(\left\{(u, v) \in \mathbb{T}^{2}: 0 \leqslant u, v \leqslant \frac{1}{2},-2(1-\epsilon) \frac{\epsilon}{3} \leqslant v-u \leqslant \frac{\epsilon}{3}\right\}\right) .
$$

Claim B.2 We have $H_{\epsilon, 2}^{-1}\left(\mathcal{C}_{\epsilon}\right) \subset \mathcal{C}_{\epsilon}$ for all $\epsilon<\frac{1}{2}$.

Proof. A strip around the diagonal can be defined as $\left\{(u, v) \in \mathbb{T}^{2}:-a \leqslant v-u \leqslant b\right\}$ for some pair $(a, b)$ of non-negative numbers. The boundaries of $\mathcal{C}_{\epsilon}$ above result from the following considerations. We want $\mathcal{C}_{\epsilon}$ to only have pre-images in $\mathcal{I}_{1} \cup \mathcal{I}_{1}+\left(\frac{1}{2}, \frac{1}{2}\right)$ and we want all these pre-images to be included in $\mathcal{C}_{\epsilon}$.

The first constraint imposes that the part of $\mathcal{C}_{\epsilon}$ that lies in the lower left quadrant $\{(u, v): 0 \leqslant$ $\left.u, v \leqslant \frac{1}{2}\right\}$ be the largest strip around the diagonal that does not intersect the dark grey region in Fig. 9. This part writes

$$
\mathcal{C}_{\text {part }}=\left\{(u, v) \in \mathbb{T}^{2}: 0 \leqslant u, v \leqslant \frac{1}{2},-a \leqslant v-u \leqslant b\right\},
$$

with $a \leqslant \epsilon$ and $b \leqslant \frac{\epsilon}{3}$. Now, pre-images of points in this set that are located in the lower left quadrant write $\left(\frac{u}{2(1-\epsilon)}, \frac{v}{2(1-\epsilon)}\right)$; hence they obviously belong to $\mathcal{C}_{\text {part }}$. Moreover, those pre-images located in the upper right quadrant - which write $\left(\frac{u}{2(1-\epsilon)}, \frac{v}{2(1-\epsilon)}\right)+\left(\frac{1}{2}, \frac{1}{2}\right)$ - must be contained in

$$
S_{v} \circ S_{u}\left(\mathcal{C}_{\text {part }}\right)=\left\{(u, v) \in \mathbb{T}^{2}: \frac{1}{2} \leqslant u, v \leqslant 1,-b \leqslant v-u \leqslant a\right\}
$$

Therefore the following inequalities must be satisfied: $b \leqslant 2(1-\epsilon) a$ and $a \leqslant 2(1-\epsilon) b$. Computing the maximal coordinates $(a, b)$ that simultaneously satisfy the four inequalities, we obtain $a=2(1-\epsilon) \frac{\epsilon}{3}$ and $b=\frac{\epsilon}{3}$. Applying the symmetries $S_{u}$ and $S_{v}$ then gives the desired conclusion.

\footnotetext{
${ }^{13}$ One checks that $H_{\epsilon, 2}\left(O\left(\mathcal{P}_{\epsilon}\right)\right) \cap\left(\mathcal{I}_{1} \cup \mathcal{I}_{1}+\left(\frac{1}{2}, \frac{1}{2}\right)\right) \subset \mathcal{C}_{\epsilon}$.
} 
As before, since the only orbits that may never leave $\mathcal{C}_{\epsilon}$ are those contained in the diagonal and the anti-diagonal $\left\{(u,-u)(\bmod 1): u \in \mathbb{T}^{1}\right\}$, the set $\mathcal{C}_{\epsilon}$ cannot be part of the Milnor attractor, viz. $\mathcal{A}_{\epsilon} \backslash\left(\mathcal{B}_{\epsilon} \cup \mathcal{C}_{\epsilon}\right)$, see Fig. 10. Besides, later on, we shall need the following comment in order to evaluate the structure of the resulting set

Remark B.3 The intersection, in the lower left quadrant, of the lower boundary $v=u-2(1-\epsilon) \frac{\epsilon}{3}$ of $\mathcal{C}_{\epsilon}$ with the right boundary $u+v=1-\epsilon$ of $H_{\epsilon, 2}(\mathrm{I})$, lies above the horizontal line $v=\frac{1}{4}$ iff $\epsilon<\frac{5-\sqrt{13}}{4}$.

One can now consider the image of the remaining part of the central strip $\mathcal{S}_{\epsilon}$, namely

$$
\mathcal{D}_{\epsilon}=H_{\epsilon, 2}\left(\mathcal{S}_{\epsilon} \cap\left(\mathcal{I}_{2} \cup \mathcal{I}_{3}\right)\right) .
$$

The set $\mathcal{D}_{\epsilon}$ consists of two triangles that are symmetric around the vertical axis $\{0(\bmod 1)\} \times \mathbb{T}^{1}$ (Fig. 10) and we have the following property.

Claim B.4 $H_{\epsilon, 2}^{-1}\left(\mathcal{D}_{\epsilon}\right) \subset \mathcal{B}_{\epsilon} \cup \mathcal{C}_{\epsilon} \cup \mathcal{D}_{\epsilon} \cup\left(\mathbb{T}^{2} \backslash \mathcal{A}_{\epsilon}\right)$ for all $\epsilon<\frac{1}{2}$.

No orbit can stay forever in $\mathcal{D}_{\epsilon}$. Hence, the Milnor attractor must be contained in $\mathcal{A}_{\epsilon} \backslash\left(\mathcal{B}_{\epsilon} \cup \mathcal{C}_{\epsilon} \cup \mathcal{D}_{\epsilon}\right)$, see Figure 11.
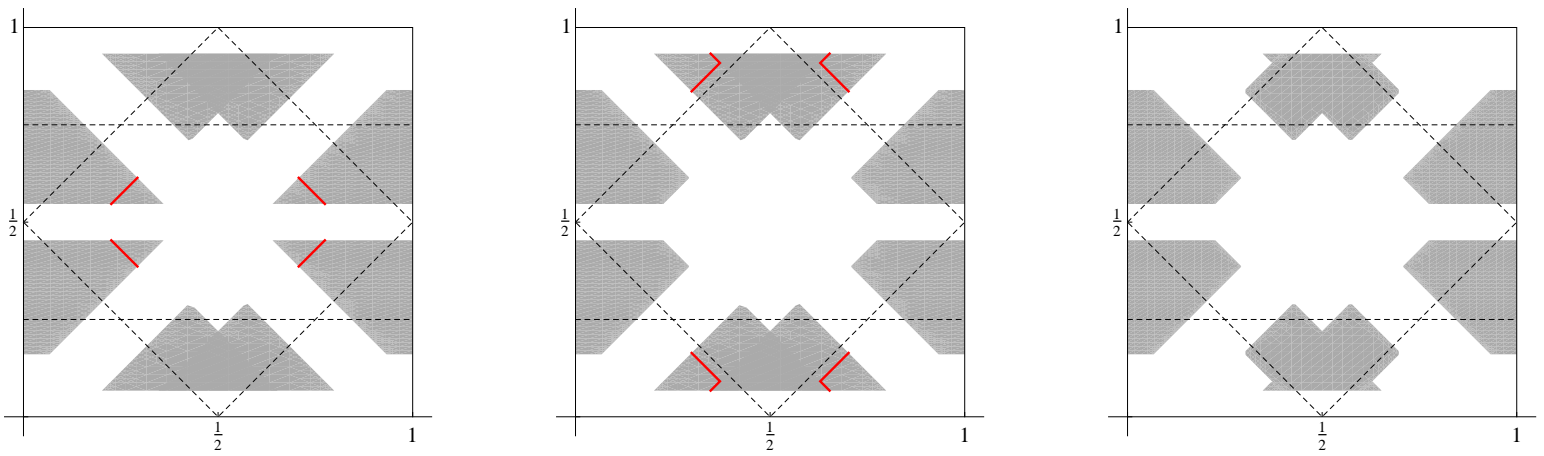

Figure 11: Construction of the Milnor attractor continued from Fig. 10. Left panel. The set $\mathcal{A}_{\epsilon} \backslash\left(\mathcal{B}_{\epsilon} \cup \mathcal{C}_{\epsilon} \cup \mathcal{D}_{\epsilon}\right)$ (grey region) and boundaries (red lines) of the four triangles $\mathcal{E}_{\epsilon}=O\left(\mathcal{E}_{\text {part }}\right)$. Center panel. The set $\mathcal{A}_{\epsilon} \backslash\left(\mathcal{B}_{\epsilon} \cup \mathcal{C}_{\epsilon} \cup \mathcal{D}_{\epsilon} \cup \mathcal{E}_{\epsilon}\right)$ (grey region) and boundaries (red lines) of the quadrilaterals $\mathcal{F}_{\epsilon}$. Right panel. The final set $\mathcal{A}_{\epsilon} \backslash\left(\mathcal{B}_{\epsilon} \cup \mathcal{C}_{\epsilon} \cup \mathcal{D}_{\epsilon} \cup \mathcal{E}_{\epsilon} \cup \mathcal{F}_{\epsilon}\right)$ for $\epsilon<\frac{5-\sqrt{13}}{4} \sim 0.349$.

Proof of the Claim. Consider for simplicity the triangle

$$
D_{\text {part }}=H_{\epsilon, 2}\left(\mathcal{S}_{\epsilon} \cap S_{v}(\mathrm{II})\right)=\left\{(u, v) \in \mathbb{T}^{2}: \frac{\epsilon}{3} \leqslant v-u, v \leqslant(2-\epsilon) \frac{\epsilon}{3}\right\},
$$

the properties of other parts of $\mathcal{D}_{\epsilon}$ will follow from symmetries. As its expression indicates, the set $D_{\text {part }}$ has four pre-images. One is obviously $\mathcal{S}_{\epsilon} \cap S_{v}$ (II) which is a subset of $\mathbb{T}^{2} \backslash \mathcal{A}_{\epsilon}$. By translation invariance, another one is $\left(\mathcal{S}_{\epsilon} \cap S_{v}(\mathrm{II})\right)+\left(\frac{1}{2}, \frac{1}{2}\right)$ which is a subset of $\mathcal{B}_{\epsilon}$. A third pre-image is obtained by the pure contraction $\left.(2(1-\epsilon))^{-1} \mathrm{Id}\right|_{\mathbb{T}^{2}}$; hence it is contained in $D_{\text {part }} \cup \mathcal{C}_{\epsilon}$. The last pre-image is the triangle

$$
\left\{(u, v) \in \mathbb{T}^{2}: \frac{1}{2} \leqslant u, \frac{\epsilon}{6(1-\epsilon)} \leqslant v-u, v \leqslant \frac{1}{2}+\frac{(2-\epsilon) \epsilon}{6(1-\epsilon)}\right\} .
$$

Explicit calculations show that this set is included in the union of the upper right pentagon $\mathcal{P}_{\epsilon}$ with $\mathcal{C}_{\epsilon}$. 
Remark B.5 The upper (horizontal) boundary $v=(2-\epsilon) \frac{\epsilon}{3}$ of the triangle $D_{\text {part }}$ lies below the line $v=\frac{1}{4}$ for all $\epsilon \in\left(0, \frac{1}{2}\right)$.

Additional restrictions on the attractor can be obtained by determining the regions for which all pre-images belong to $\mathcal{A}_{\epsilon} \backslash\left(\mathcal{B}_{\epsilon} \cup \mathcal{C}_{\epsilon}\right)$. The construction of $\mathcal{C}_{\epsilon}$ suggests the image of a neighborhood of the point $\left(\frac{1}{4}, \frac{1}{4}\right) \in$ II (and its symmetric images) as a good candidate. Optimizing this set yields the following conclusion. Let the following triangle located in the lower right part of the image triangle $H_{\epsilon, 2}(\mathrm{II})$, see Fig. 9

$$
\mathcal{E}_{\text {part }}=\left\{(u, v) \in H_{\epsilon, 2}(\mathrm{II}): v-u \leqslant 2(2-\epsilon) \frac{\epsilon}{3}\right\}
$$

and let $\mathcal{E}_{\epsilon}=O\left(\mathcal{E}_{\text {part }}\right)$.

Claim B.6 We have $H_{\epsilon, 2}^{-1}\left(\mathcal{E}_{\epsilon}\right) \subset \mathcal{C}_{\epsilon}$ for all $\epsilon<\frac{1}{2}$.

Proof. A candidate for $\mathcal{E}_{\text {part }}$ can be defined as $\left\{(u, v) \in H_{\epsilon, 2}(\mathrm{II}): v-u \leqslant a\right\}$ for some $a \geqslant 0$; the constraint $v-u \leqslant a$ results from requiring that the upper left boundary of the triangle be parallel to some boundaries of $\mathcal{C}_{\epsilon}$.

Now, the value of $a$ that defines $\mathcal{E}_{\text {part }}$ is obtained from the following considerations. We want $\mathcal{E}_{\text {part }}$ to only have pre-images in $\mathrm{II} \cap \mathcal{C}_{\epsilon}$ and in $\left(\mathrm{II}+\left(\frac{1}{2}, \frac{1}{2}\right)\right) \cap \mathcal{C}_{\epsilon}$.

The constraint that $\mathcal{E}_{\text {part }}$ has only two pre-images imposes that this set does not intersect the dark grey region of $H_{\epsilon, 2}$ (II) in Fig. 9. Associated explicit calculations yield $a \leqslant \frac{4 \epsilon}{3}$. Furthermore, the constraint that the pre-image in II belongs to $\mathcal{C}_{\epsilon}$ is clearly stronger than requiring that the pre-image in II $+\left(\frac{1}{2}, \frac{1}{2}\right)$ belongs to $\mathcal{C}_{\epsilon}$ (because the strip above the diagonal that composes $\mathcal{C}_{\epsilon}$ is narrower in the former region than in the latter). Explicit calculations on pre-images show that this constraint yields the inequality $a \leqslant 2(2-\epsilon) \frac{\epsilon}{3}$. The Claim then follows from the fact that $2(2-\epsilon) \frac{\epsilon}{3} \leqslant \frac{4 \epsilon}{3}$ which holds for all $\epsilon \geqslant 0$.

Remark B.7 The left boundary $v=u+2(2-\epsilon) \frac{\epsilon}{3}$ of $\mathcal{E}_{\text {part }}$ lies below the inclined line $v=u+\frac{1}{2}$ for all $\epsilon \in\left(0, \frac{1}{2}\right)$.

Repeating the same strategy, we observe that the lowest left corner of the triangle $S_{u}\left(\mathcal{E}_{\text {part }}\right)$ is mapped to the point $\left((1-\epsilon) \epsilon, \frac{(1-\epsilon) \epsilon}{3}\right)$ that lies at the intersection of the boundaries of $\mathcal{B}_{\epsilon}$ and $\mathcal{C}_{\epsilon}$ in I. Hence, any point in a sufficiently small neighborhood of this point has only two pre-images: one inside $\mathcal{B}_{\epsilon} \cup \mathcal{C}_{\epsilon}$ and the other one belongs to the triangle $S_{u}\left(\mathcal{E}_{\text {part }}\right)$. Consequently, any such neigborhood cannot be recurrent. By computing the largest set with that property, we obtain the following quadrilateral (Fig. 11)

$$
\mathcal{F}_{\text {part }}=\left\{(u, v) \in \mathbb{T}^{2}:(1-\epsilon) \frac{\epsilon}{3} \leqslant v,-\epsilon \leqslant v-u \leqslant-2(1-\epsilon) \frac{\epsilon}{3}, u+v \leqslant 4(1-\epsilon)(2-\epsilon) \frac{\epsilon}{3}\right\}
$$

and the following conclusion (whose proof is left to the reader)

Claim B.8 Let $\mathcal{F}_{\epsilon}=O\left(\mathcal{F}_{\text {part }}\right)$. We have $H_{\epsilon, 2}^{-1}\left(\mathcal{F}_{\epsilon}\right) \subset \mathcal{B}_{\epsilon} \cup \mathcal{C}_{\epsilon} \cup \mathcal{E}_{\epsilon}$ for all $\epsilon<\frac{1}{2}$.

Remark B.9 The line $u+v=4(1-\epsilon)(2-\epsilon) \frac{\epsilon}{3}$ lies below the line $u+v=\frac{1}{2}$ iff $\epsilon<\frac{5-\sqrt{13}}{4}$. Under the same condition, the intersection of the lines $v=u-\epsilon$ and $v=(1-\epsilon) \frac{\epsilon}{3}$ lies below the line $u+v=\frac{1}{2}$.

Altogether, the information collected in this appendix is summarized in the following statement. 
Proposition B.10 For every $\epsilon \in\left(0, \frac{1}{2}\right)$, the Milnor attractor of the map $H_{\epsilon, 2}$ must be contained in the set $\mathcal{M}_{\epsilon}$ defined by

$$
\mathcal{M}_{\epsilon}=\mathcal{A}_{\epsilon} \backslash\left(\mathcal{B}_{\epsilon} \cup \mathcal{C}_{\epsilon} \cup \mathcal{D}_{\epsilon} \cup \mathcal{E}_{\epsilon} \cup \mathcal{F}_{\epsilon}\right)(\bmod 0) .
$$

Moreover, we have

$$
H_{\epsilon, 2}\left(\mathcal{M}_{\epsilon}\right)=\mathcal{M}_{\epsilon}(\bmod 0),
$$

and the restriction of this set to the lower left quadrant of the fundamental domain writes

$$
\begin{aligned}
\mathcal{M}_{\epsilon} \cap\left\{(u, v): 0 \leqslant u, v \leqslant \frac{1}{2}\right\} & =\bigcup_{i=1}^{6} P_{i, \epsilon}(\bmod 0) \text { if } \epsilon<\frac{5-\sqrt{13}}{4} \\
& =\bigcup_{i=1}^{3} P_{i, \epsilon} \cup P_{5, \epsilon}(\bmod 0) \text { if } \epsilon \geqslant \frac{5-\sqrt{13}}{4}
\end{aligned}
$$

(in particular the sets $P_{4, \epsilon}$ and $P_{6, \epsilon}$ are empty in the second case) where the polygons $\left\{P_{i, \epsilon}\right\}_{i=1}^{6}$ on which $H_{\epsilon, 2}$ is continuous are shown in Fig. 12.
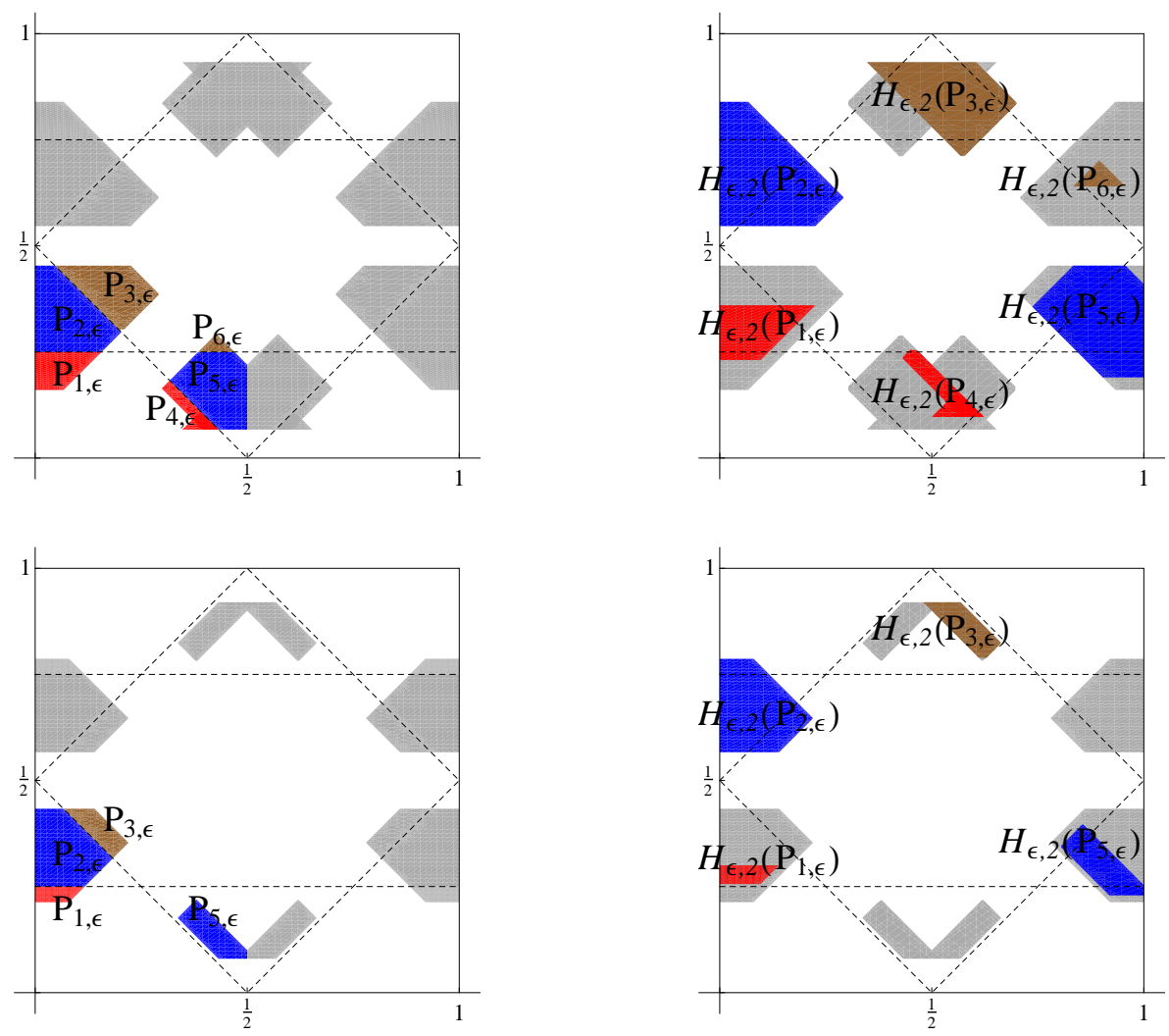

Figure 12: Decomposition of the set $\mathcal{M}_{\epsilon}$ into continuity atom in each quadrant (left panel) and representation of their image (right panel). Top: $\epsilon=0.28<\frac{5-\sqrt{13}}{4}$. Bottom $\epsilon=0.4>\frac{5-\sqrt{13}}{4}$.

Analytic expressions for the boundaries of the polygons $\left\{\mathrm{P}_{i, \epsilon}\right\}_{i=1}^{6}$ can be easily deduced from the previous analysis. As is always the case in the paper, those boundaries that are composed of pieces of discontinuities lines are excluded of these sets.

Sketch of proof. That the Milnor attractor must be contained in $\mathcal{M}_{\epsilon}$ is a consequence of the construction in this appendix. The existence of the polygons $\mathrm{P}_{i, \epsilon}$ is granted by the Remarks after 
proofs, especially Remarks B.3 and B.9. Finally, one checks that the construction implies the following relations (Fig. 12)

$$
H_{\epsilon, 2}\left(\mathrm{P}_{1, \epsilon}\right)=S_{v}\left(\bigcup_{i=1}^{3} \mathrm{P}_{i, \epsilon}\right)(\bmod 0)
$$

and

$$
\begin{aligned}
H_{\epsilon, 2}\left(\mathrm{P}_{3, \epsilon} \cup S_{u}\left(\mathrm{P}_{3, \epsilon}\right)\right) & =S_{v}\left(\bigcup_{i=4}^{6} \bigcup_{k=0,1} S_{u}^{k}\left(\mathrm{P}_{i, \epsilon}\right)\right)(\bmod 0) \text { if } \epsilon<\frac{5-\sqrt{13}}{4} \\
& =S_{v}\left(\mathrm{P}_{5, \epsilon} \cup S_{u}\left(\mathrm{P}_{5, \epsilon}\right)\right) \text { if } \epsilon \geqslant \frac{5-\sqrt{13}}{4}
\end{aligned}
$$

which, together with symmetries, imply $H_{\epsilon, 2}\left(\mathcal{M}_{\epsilon}\right)=\mathcal{M}_{\epsilon}$.

\section{B.2 Symmetry breaking for $\mathcal{M}_{\epsilon}$, proof of Proposition 4.4}

\section{B.2.1 Decomposition of $\mathcal{M}_{\epsilon}$ into two asymmetric invariant sets}

Throughout this section, we assume $\epsilon \geqslant \frac{5-\sqrt{13}}{4}$ so that the sets $\mathrm{P}_{4, \epsilon}$ and $\mathrm{P}_{6, \epsilon}$ are empty. Using that $\frac{4-\sqrt{10}}{2}>\frac{5-\sqrt{13}}{4}$, the inequality $\epsilon>\frac{4-\sqrt{10}}{2}$ in Proposition 4.4 is equivalent to the condition $\mathrm{P}_{5, \epsilon}$ does not intersect the vertical axis $\left\{\frac{1}{2}\right\} \times \mathbb{T}^{1}$ (i.e. we have $\mathrm{P}_{5, \epsilon} \cap S_{u}\left(\mathrm{P}_{5, \epsilon}\right)=\emptyset$ ). In this case, the previous construction of $\mathcal{M}_{\epsilon}$ implies (Fig. 12, bottom panels)

$$
H_{\epsilon, 2}\left(\mathrm{P}_{3, \epsilon}\right)=S_{u} \circ S_{v}\left(\mathrm{P}_{5, \epsilon}\right)
$$

In addition, we have

$$
H_{\epsilon, 2} \circ S_{u}\left(\mathrm{P}_{5, \epsilon}\right) \subset \bigcup_{i=1}^{3} \mathrm{P}_{i, \epsilon}(\bmod 0)
$$

Let

$$
\mathcal{N}_{\epsilon}=\bigcup_{k=0,1} S_{v}^{k}\left(\bigcup_{i=1}^{3} \mathrm{P}_{i, \epsilon} \cup S_{u}\left(\mathrm{P}_{5, \epsilon}\right)\right)(\bmod 0) .
$$

Then we have $\mathcal{M}_{\epsilon}=\mathcal{N}_{\epsilon} \cup S_{u}\left(\mathcal{N}_{\epsilon}\right)$ and the arguments above imply $\mathcal{N}_{\epsilon}(\bmod 0)$ is invariant under $H_{\epsilon, 2}$ when $\epsilon>\frac{4-\sqrt{10}}{2}$. The multiplicity entropy argument implies that the restriction $\left.H_{\epsilon, 2}\right|_{\mathcal{N}_{\epsilon}}$ must have an a.c.i.m. whose density is uniformly positive on an open ball.

This proves that, when $\epsilon>\frac{4-\sqrt{10}}{2}, H_{\epsilon, 2}$ has at least two Lebesgue ergodic components and every ergodic component must break the symmetry $S_{u}$, and therefore must break the symmetry $S_{u} \circ S_{v}$. Applying the inverse change of coordinates $M^{-1}$, the same conclusion holds for $G_{\epsilon, 2}$. The first part of Proposition 4.4 is proved.

\section{B.2.2 Existence of six Lebesgue ergodic components}

Let $\epsilon^{*}$ be the unique real root of the polynomial $12 \epsilon^{3}-34 \epsilon^{2}+33 \epsilon-9$. Numerical calculations show that $\epsilon^{*} \geqslant 0.444$.

Claim B.11 For every $\epsilon>\epsilon^{*}$, there exists $\frac{1}{4}<a(\epsilon)<b(\epsilon)<\frac{1}{2}-\frac{\epsilon}{6}$ such that 
- the union set

$$
\mathcal{Q}_{1, \epsilon}=\bigcup_{t=0}^{2} H_{\epsilon, 2}^{t}\left(\left\{(u, v) \in \bigcup_{i=1}^{3} P_{i, \epsilon}: v \leqslant a(\epsilon)\right\}\right),
$$

defines a forward invariant set, asymmetric with respect to both $S_{u}$ and $S_{v}$, viz.

$$
H_{\epsilon, 2}\left(\mathcal{Q}_{1, \epsilon}\right) \subset \mathcal{Q}_{1, \epsilon}(\bmod 0) \text { and } S_{u}\left(\mathcal{Q}_{1, \epsilon}\right) \cap \mathcal{Q}_{1, \epsilon}=S_{v}\left(\mathcal{Q}_{1, \epsilon}\right) \cap \mathcal{Q}_{1, \epsilon}=\emptyset .
$$

- the union set

$$
\mathcal{Q}_{2, \epsilon}=\bigcup_{t=0}^{2} \bigcup_{k=0,1} H_{\epsilon, 2}^{t} \circ S_{v}^{k}\left(\left\{(u, v) \in \bigcup_{i=1}^{3} P_{i, \epsilon}: a(\epsilon) \leqslant v \leqslant b(\epsilon)\right\}\right),
$$

defines a forward invariant $S_{u}$-asymmetric set, viz.

$$
H_{\epsilon, 2}\left(\mathcal{Q}_{2, \epsilon}\right) \subset \mathcal{Q}_{2, \epsilon}(\bmod 0) \text { and } S_{u}\left(\mathcal{Q}_{2, \epsilon}\right) \cap \mathcal{Q}_{2, \epsilon}=\emptyset .
$$

As argued in the previous section, each set $\mathcal{Q}_{1, \epsilon}$ and $\mathcal{Q}_{2, \epsilon}$ must contain a Lebesgue ergodic component of $H_{\epsilon, 2}$. Applying $S_{u}$ and $S_{v}$, we conclude that there must be at least six such components. The proof of Proposition 4.4 is complete.

Proof of the Claim. Given $a>\frac{1}{4}$, let

$$
\mathrm{Q}_{a}=\left\{(u, v) \in \bigcup_{i=1}^{3} \mathrm{P}_{i, \epsilon}: v \leqslant a\right\} .
$$

We are going to determine the quantity $a(\epsilon)$ such that the set $\bigcup_{t=0}^{2} H_{\epsilon, 2}^{t}\left(\mathrm{Q}_{a(\epsilon)}\right)$ be forward invariant. This requires considering the first two images of $\mathrm{Q}_{a}$.

The discontinuity line $u+v=\frac{1}{2}$ meets the lower boundary $v=u+\frac{\epsilon}{3}$ of $\bigcup_{i=1}^{3} \mathrm{P}_{i, \epsilon}$ at a point with vertical coordinate $v=\frac{1}{4}+\frac{\epsilon}{6}$. Hence, if $a \leqslant \frac{1}{4}+\frac{\epsilon}{6}$, we are sure that $\mathrm{Q}_{a} \cap \mathrm{P}_{3, \epsilon}=\emptyset$. Moreover, we have

$$
H_{\epsilon, 2}\left(\mathrm{P}_{1, \epsilon}\right) \subset\left\{(u, v) \in \bigcup_{i=1}^{3} \mathrm{P}_{i, \epsilon}: v \leqslant \frac{1-\epsilon}{2}\right\}(\bmod 0) .
$$

We have $\mathrm{Q}_{a} \cap \mathrm{P}_{1, \epsilon}=\mathrm{P}_{1, \epsilon}$ since $a>\frac{1}{4}$. Hence, the condition $\frac{1-\epsilon}{2} \leqslant a$ implies $H_{\epsilon, 2}\left(\mathrm{Q}_{a} \cap \mathrm{P}_{1, \epsilon}\right) \subset \mathrm{Q}_{a}$ and we are left with the analysis of

$$
\mathrm{Q}_{a}^{1}=H_{\epsilon, 2}\left(\mathrm{Q}_{a} \cap \mathrm{P}_{2, \epsilon}\right)=\left\{(u, v) \in \bigcup_{i=1}^{3} S_{v}\left(\mathrm{P}_{i, \epsilon}\right): v \leqslant a_{2}\right\}(\bmod 0)
$$

where $a_{2}=2(1-\epsilon) a+\frac{2 \epsilon}{3}$. Assuming $a_{2} \leqslant \frac{3}{4}$, then $\mathrm{Q}_{a}^{1}$ does not intersect $S_{v}\left(\mathrm{P}_{1, \epsilon}\right)$. Moreover

$$
H_{\epsilon, 2}\left(\mathrm{Q}_{a}^{1} \cap S_{v}\left(\mathrm{P}_{2, \epsilon}\right)\right)=\left\{(u, v) \in \bigcup_{i=1}^{3} \mathrm{P}_{i, \epsilon}: v \leqslant a_{3}\right\}(\bmod 0),
$$

where $a_{3}=2(1-\epsilon) a_{2}-1+\frac{4 \epsilon}{3}$. Therefore, under the condition $a_{3} \leqslant a$ (which turns out to be equivalent to $a \leqslant \frac{1}{3}$ ), we have

$$
H_{\epsilon, 2}\left(\mathrm{Q}_{a}^{1} \cap S_{v}\left(\mathrm{P}_{2, \epsilon}\right)\right) \subset \mathrm{Q}_{a}(\bmod 0) .
$$


On the other hand, using $\epsilon>\frac{4-\sqrt{10}}{2}$ we obtain

$$
H_{\epsilon, 2}\left(\mathrm{Q}_{a}^{1} \cap S_{v}\left(\mathrm{P}_{3, \epsilon}\right)\right) \subset\left\{(u, v) \in S_{u}\left(\mathrm{P}_{5, \epsilon}\right): v \leqslant a_{4}\right\},
$$

where $a_{4}=2(1-\epsilon) a_{2}-(1-\epsilon)$, and then

$$
H_{\epsilon, 2}^{2}\left(\mathrm{Q}_{a}^{1} \cap S_{v}\left(\mathrm{P}_{3, \epsilon}\right)\right) \subset\left\{(u, v) \in \bigcup_{i=1}^{3} \mathrm{P}_{i, \epsilon}: v \leqslant a_{5}\right\}(\bmod 0),
$$

where $a_{5}=2(1-\epsilon) a_{4}+\frac{\epsilon}{3}$. Obviously, if $a_{5} \leqslant a$, then this set is included in the original set $\mathrm{Q}_{a}$. Moreover, provided that all inequalities above hold, the reasoning here implies $\bigcup_{t=0}^{2} H_{\epsilon, 2}^{t}\left(\mathrm{Q}_{a}\right) \subset$ $\mathrm{Q}_{a}(\bmod 0)$ as desired. In order to ensure that $\mathrm{Q}_{a}$ breaks the $S_{v^{-} \text {-symmetry, }}{ }^{14}$ one needs to make sure that the inequality $a_{2} \leqslant 1-a$ holds.

To conclude the proof of the first item, we need to compute the value of $a$ and check all conditions. Solving the equation $a_{5}(a)=a$ yields $a(\epsilon)=\frac{6-9 \epsilon+4 \epsilon^{2}}{3\left(7-10 \epsilon+4 \epsilon^{2}\right)}$. Moreover, one checks that

$$
\frac{1}{4}<a(\epsilon)<\min \left\{\frac{1}{4}+\frac{\epsilon}{6}, \frac{1}{3}\right\} \text { if } \frac{4-\sqrt{10}}{2}<\epsilon<\frac{1}{2} .
$$

Now, the inequality $\frac{1-\epsilon}{2} \leqslant a(\epsilon)$ is equivalent to $12 \epsilon^{3}-34 \epsilon^{2}+33 \epsilon-9 \geqslant 0$ and this polynomial has a single real root $\epsilon^{*}$. Finally one also check that the following holds

$$
a_{2}(a(\epsilon)) \leqslant \min \left\{\frac{3}{4}, 1-a(\epsilon)\right\} \text { if } 0<\epsilon<\frac{1}{2},
$$

and the proof of the first item is complete.

We proceed similarly to prove the second item. Given $a(\epsilon)<b<\frac{1}{2}-\frac{\epsilon}{6}$, consider the set ${ }^{15}$

$$
\bigcup_{k=0,1} S_{v}^{k}\left(\mathrm{Q}_{b}\right) \text { where } \mathrm{Q}_{b}=\left\{(u, v) \in \bigcup_{i=2,3} \mathrm{P}_{i, \epsilon}: a(\epsilon) \leqslant v \leqslant b\right\} .
$$

By symmetry it suffices to consider the images of $\mathrm{Q}_{b}$. We want the part of this set that intersects $\mathrm{P}_{2, \epsilon}$ to be mapped inside $S_{v}\left(\mathrm{Q}_{b}\right)$. This is equivalent to the inequalities $1-b \leqslant a_{2}(\epsilon)$ and $b_{2} \leqslant 1-a(\epsilon)$ where $b_{2}=2(1-\epsilon) b+\frac{2 \epsilon}{3}$.

Moreover, the part of $\mathrm{Q}_{b}$ that intersects $\mathrm{P}_{3, \epsilon}$ is mapped into $S_{v} \circ S_{u}\left(\mathrm{P}_{5, \epsilon}\right)$ and then into $\bigcup_{i=1}^{3} S_{v}\left(\mathrm{P}_{i, \epsilon}\right)(\bmod 0)$. When this happens, we want this image to sit in $S_{v}\left(\mathrm{Q}_{b}\right)$. This imposes two inequalities. One inequality is $b_{4} \leqslant 1-a(\epsilon)$ where $b_{4}=2(1-\epsilon) b_{3}-1+\frac{5 \epsilon}{3}$ and $b_{3}=2(1-\epsilon) b+\epsilon$.

In order to get the second inequality, we observe that the part of $\mathrm{Q}_{b}$ that intersects $\mathrm{P}_{3, \epsilon}$ must obey $v \geqslant v(\epsilon)=\frac{1}{4}+\frac{\epsilon}{6}$. Hence, the image in $S_{v} \circ S_{u}\left(\mathrm{P}_{5, \epsilon}\right)$ has to satisfy $v \geqslant v_{2}(\epsilon)=2(1-\epsilon) v(\epsilon)+\epsilon$ and the second desired inequality is $2(1-\epsilon) v_{2}(\epsilon)-1+\frac{5 \epsilon}{3} \geqslant 1-b$.

Solving $b_{4}(b)=1-a(\epsilon)$ for $b$ yields $b(\epsilon)=\frac{9-14 \epsilon+6 \epsilon^{2}}{3\left(7-10 \epsilon+4 \epsilon^{2}\right)}$. Now one checks that, for all $\epsilon<\frac{1}{2}$, we have $b(\epsilon)<\frac{1}{2}-\frac{\epsilon}{6}$ and

$$
\max \left\{2(1-\epsilon) b(\epsilon)+\frac{2 \epsilon}{3}, 2(1-\epsilon)(2(1-\epsilon) b(\epsilon)+\epsilon)-1+\frac{5 \epsilon}{3}\right\}<1-a(\epsilon) .
$$

\footnotetext{
${ }^{14}$ By construction, it already breaks the $S_{u}$-symmetry.

${ }^{15}$ The assumption $v \geqslant a(\epsilon)$ prevents $\mathrm{Q}_{b}$ to intersect the set $\mathrm{P}_{1, \epsilon}$.
} 
Under the same condition, the first inequality below holds

$$
b(\epsilon) \geqslant \max \left\{a(\epsilon), 2-2(1-\epsilon)(2(1-\epsilon) v(\epsilon)+\epsilon)-\frac{5 \epsilon}{3}\right\} .
$$

One checks that the second inequality holds provided that $0.4<\epsilon<\frac{1}{2}$. The second item is proved.

\section{B.3 L.e.o. property, proof of transitivity in Proposition 4.3}

Recall that a mapping $F$ defined on some topological space $X$ (endowed with some $\sigma$-algebra and Borel measure) is said to be locally eventually onto (l.e.o.) if for every open set $U \subset X$, there exists $t \in \mathbb{N}$ such that $F^{t}(U)=X\left(\right.$ or $F^{t}(U)=X(\bmod 0)$ ). Every l.e.o. map must be transitive. Accordingly, the transitivity claim for $H_{\epsilon, 2}$ in $\mathcal{M}_{\epsilon}$, as stated in Proposition 4.3 of section 4.2, is a consequence of the following result.

Proposition B.12 For every $\epsilon<1-\frac{\sqrt{2}}{2}$, the map $\left.H_{\epsilon, 2}\right|_{\mathcal{M}_{\epsilon}}$ is l.e.o.

This section is devoted to the proof of this Proposition which consists in proving the following statements $^{16}$

(a) For every $\epsilon<1-\frac{\sqrt{2}}{2}$ and every open set $U \subset \mathcal{M}_{\epsilon}$, there exists $t \in \mathbb{N}$ such that $H_{\epsilon, 2}^{t}(U)$ contains a $S_{u}$-invariant square.

(b) For every $\epsilon<1-\frac{\sqrt{2}}{2}$ and every $S_{u}$-invariant square $U \subset \mathcal{M}_{\epsilon}$, there exists $t \in \mathbb{N}$ such that $H_{\epsilon, 2}^{t}(U)=\mathcal{M}_{\epsilon}(\bmod 0)$.

For convenience, we prove the second statement before the first one.

\section{B.3.1 Proof of statement (b)}

Let $\epsilon<1-\frac{\sqrt{2}}{2}$ be arbitrary. (Notice that $1-\frac{\sqrt{2}}{2}<\frac{5-\sqrt{13}}{4}$; hence $\mathrm{P}_{4, \epsilon}$ and $\mathrm{P}_{6, \epsilon}$ are non-empty.) The images of the atoms of the partition of $\mathcal{M}_{\epsilon}$, especially of $P_{2, \epsilon}$ and $P_{3, \epsilon}$ (see Fig. 12) indicate that, in order to show that an iterate of a $S_{u}$-invariant square covers $\mathcal{M}_{\epsilon}(\bmod 0)$, it suffices to ensure that an (earlier) iterate covers either the set $P_{2, \epsilon} \cup S_{u}\left(P_{2, \epsilon}\right)$ or its symmetric image $S_{v}\left(P_{2, \epsilon} \cup S_{u}\left(P_{2, \epsilon}\right)\right)$.

The proof relies on the following properties

- The image of any $S_{u}$-invariant rectangle $U \subset \mathcal{M}_{\epsilon}$ under $H_{\epsilon, 2}$ consists of either one or two $S_{u}$-invariant rectangles.

- The horizontal width of any $S_{u}$-invariant rectangle in $\mathcal{M}_{\epsilon}$ is dilated by a factor $2(1-\epsilon)$ under the action of $H_{\epsilon, 2}$.

- Let $\mathcal{J}=\left(\frac{1}{4}, \frac{1}{2}-\frac{\epsilon}{6}\right)$ be defined by

$$
\left(P_{2, \epsilon} \cup S_{u}\left(P_{2, \epsilon}\right)\right) \cap\{0(\bmod 1)\} \times \mathbb{T}^{1}=\{0(\bmod 1)\} \times \mathcal{J} .
$$

\footnotetext{
${ }^{16}$ The term $S_{u}$-invariant square (resp. $S_{u}$-invariant rectangle) denotes any square (resp. rectangle) in $\mathbb{T}^{2}$ which the vertical axis $\{0(\bmod 1)\} \times \mathbb{T}^{1}$ separates into two rectangles that are image one another under the action of $S_{u}$.
} 
If an $S_{u}$-invariant rectangle $U \subset \mathcal{M}_{\epsilon}$ is such that

$$
U \cap\{0(\bmod 1)\} \times \mathbb{T}^{1}=\{0(\bmod 1)\} \times \mathcal{J} .
$$

Then we have

$$
H_{\epsilon, 2}(U) \cap\{0(\bmod 1)\} \times \mathbb{T}^{1}=\{0(\bmod 1)\} \times-\mathcal{J},
$$

Together with the symmetry $S_{v}$ and the fact that $H_{\epsilon, 2}$ is a piecewise affine conformal expanding map, these properties imply that for any $S_{u}$-invariant rectangle $U \subset \mathcal{M}_{\epsilon}$ satisfying (5), there exists $t \in \mathbb{N}$ such that $H_{\epsilon, 2}^{t}(U)$ covers either $P_{2, \epsilon} \cup S_{u}\left(P_{2, \epsilon}\right)$ or $S_{v}\left(P_{2, \epsilon} \cup S_{u}\left(P_{2, \epsilon}\right)\right)$.

Therefore, all we have to show is that, for any initial $S_{u}$-invariant square in $\mathcal{M}_{\epsilon}$, there exists an iterate under $H_{\epsilon, 2}$ that contains an $S_{u}$-invariant rectangle satisfiying (5) (or for which the symmetric rectangle under $S_{v}$ satisfies (5)).

To that goal, it suffices to consider the one-dimensional dynamics on the vertical axis $\{0(\bmod 1)\} \times$ $\mathbb{T}^{1}$ and to show that, for any initial arc in this set, there exists an iterate that covers either $\{0(\bmod 1)\} \times \mathcal{J}$ or $\{0(\bmod 1)\} \times-\mathcal{J}$.

The intersection of $\mathcal{M}_{\epsilon}$ with the vertical axis $\{0(\bmod 1)\} \times \mathbb{T}^{1}$ consists of the set $\mathcal{K} \times \mathbb{T}^{1}$ where

$$
\mathcal{K}=\left((2-\epsilon) \frac{\epsilon}{3}, \frac{1}{2}-\frac{\epsilon}{6}\right) \cup\left(\frac{1}{2}+\frac{\epsilon}{6}, 1-(2-\epsilon) \frac{\epsilon}{3}\right) .
$$

Let $K_{\epsilon, 2}(v)=2 v-\frac{2 \epsilon}{3}(g(v)+g(2 v))(\bmod 1)$ be the one-dimensional map defined on $\mathcal{K}$ by the restriction of $H_{\epsilon, 2}$ to $\mathcal{K} \times \mathbb{T}^{1}$. We are going to prove that $K_{\epsilon, 2}$ is l.e.o. by proceeding similarly as for Lorenz-type maps of the interval [41].

Let $I=I^{1}$ be a connected arc included in $\mathcal{K}$ and consider the following inductive process. Given $t \in \mathbb{N}$, assume that $I^{t}$ is given and let the set $I^{t+1}$ be defined by

$$
I^{t+1}=\left\{\begin{array}{cc}
K_{\epsilon, 2}\left(I^{t}\right) & \text { if }\left\{\frac{1}{4}, \frac{3}{4}\right\} \not \subset I^{t} \\
K_{\epsilon, 2}\left(\text { largest of two arcs that either } \frac{1}{4} \text { or } \frac{3}{4} \text { splits } I^{t} \text { into }\right) & \text { if }\left\{\frac{1}{4}, \frac{3}{4}\right\} \subset I^{t}
\end{array}\right.
$$

By construction, every $I^{t}$ is connected and, unless we simultaneously have $\left\{\frac{1}{4}, \frac{3}{4}\right\} \subset I^{t}$ and $\left\{\frac{1}{4}, \frac{3}{4}\right\} \subset$ $I^{t+1}$, the following inequality on arc lengths holds

$$
\left|I^{t+2}\right| \geqslant \frac{(2(1-\epsilon))^{2}}{2}\left|I^{t}\right|
$$

We have $\frac{(2(1-\epsilon))^{2}}{2}>1$ iff $\epsilon<1-\frac{\sqrt{2}}{2}$. However all sets $I^{t}$ must be contained in $\mathcal{K}$; hence both $I^{t}$ and $I^{t+1}$ must meet the discontinuity set $\left\{\frac{1}{4}, \frac{3}{4}\right\}$ for $t$ sufficiently large.

Assume w.l.o.g. that $\frac{1}{4} \in I^{t}$, the other case can be treated by applying $u \mapsto-u(\bmod 1)$. If, in addition we have $\frac{3}{4} \in I^{t+1}$, then Fig. 12 implies we must have $-\mathcal{J} \subset I^{t+1}$ as desired.

If otherwise $\frac{1}{4} \in I^{t+1}$, then it follows that $\left(\frac{1}{4}, K_{\epsilon, 2}\left(\frac{1}{4}-0\right)\right)=\left(\frac{1}{4}, \frac{1-\epsilon}{2}\right) \subset I^{t+1}$. Moreover, the arc $\left(\frac{1}{4}, \frac{1-\epsilon}{2}\right)$ contains the point $\frac{1}{3}$ when $\epsilon<1-\frac{\sqrt{2}}{2}$. However, the pair $\left\{\frac{1}{3}, \frac{2}{3}\right\}$ is a 2-periodic orbit of $K_{\epsilon, 2}$ where the first component belongs to $\mathcal{J}$ and the second one lies in $-\mathcal{J}$. Therefore, every neighborhood of $\frac{1}{3}$ contains a sufficiently long cylinder set ${ }^{17}$

$$
\mathcal{J} \cap K_{\epsilon, 2}^{-1}(-\mathcal{J}) \cap K_{\epsilon, 2}^{-2}(\mathcal{J}) \cap K_{\epsilon, 2}^{-3}(-\mathcal{J}) \cdots
$$

Consequently, for every neighborhood $U$ of $\frac{1}{3}$, there exists $t_{U} \in \mathbb{N}$ such that $K_{\epsilon, 2}^{t_{U}}(U) \supset \mathcal{J}$. Applying this statement with $U=\left(\frac{1}{4}, \frac{1-\epsilon}{2}\right) \subset I^{t+1}$, it follows that we have

$$
\text { either } \left.K_{\epsilon, 2}^{t}{ }_{\left(\frac{1}{4}, \frac{1-\epsilon}{2}\right)}^{(t+1}\right) \supset \mathcal{J} \text { or } K_{\epsilon, 2}^{t}{ }^{\left(\frac{1}{4}, \frac{1-\epsilon}{2}\right)}\left(I^{t+1}\right) \supset-\mathcal{J}
$$

as desired. The proof of statement (b) is complete.

\footnotetext{
${ }^{17}$ Every such cylinder set is non-empty because we have $K_{\epsilon, 2}(\mathcal{J}) \supset-\mathcal{J}$ and $K_{\epsilon, 2}(-\mathcal{J}) \supset \mathcal{J}$.
} 


\section{B.3.2 Proof of statement (a)}

Translation invariance $H_{\epsilon, 2}\left(u+\frac{1}{2}, v+\frac{1}{2}\right)=H_{\epsilon, 2}(u, v)$ and invariance of the vertical axis $\{0(\bmod 1)\} \times$ $\mathbb{T}^{1}$ under $H_{\epsilon, 2}$ imply

$$
H_{\epsilon, 2}\left(\left\{\frac{1}{2}\right\} \times \mathbb{T}^{1}\right)=\{0(\bmod 1)\} \times \mathbb{T}^{1} .
$$

In particular, the image under $H_{\epsilon, 2}$ of every open ball intersecting $\left\{\frac{1}{2}\right\} \times \mathbb{T}^{1}$ contains a $S_{u}$-invariant square. Accordingly, statement (a) will be an immediate consequence of the following property.

Lemma B.13 Let $\epsilon \in\left(0,1-\frac{\sqrt{2}}{2}\right)$ be given. For every open set $U \subset \mathcal{M}_{\epsilon}$ not intersecting $\{0(\bmod 1)\} \times$ $\mathbb{T}^{1}$, there exists $t_{U} \in \mathbb{N}$ such that $H_{\epsilon, 2}^{t_{U}}(U)$ contains an open ball that intersects $\left\{\frac{1}{2}\right\} \times \mathbb{T}^{1}$.

The proof is not as direct as the statement suggests. In particular, since segments can be repeatedly cut into pieces while iterating, we need to combine length considerations with arguments of area growth in order to make sure that discontinuity lines are eventually crossed in a suitable way.

Proof of the Lemma. The proof consists in establishing two claims. The first one relies on conformal expansion of $H_{\epsilon, 2}$ and deals with iterates of convex polygons whose boundaries are parallel to discontinuity lines. By this, we mean that any boundary is either an horizontal segment or a segment parallel to the diagonal $\left\{(u, u)(\bmod 1): u \in \mathbb{T}^{1}\right\}$ or to the anti-diagonal $\left\{(u,-u)(\bmod 1): u \in \mathbb{T}^{1}\right\}$. Obviously, such polygons have at most 6 edges.

Claim B.14 For any convex polygon $U$ with boundaries being parallel to discontinuity lines, and included in a single atom ${ }^{18}$ of $\mathcal{M}_{\epsilon}$, there exists $t_{U} \in \mathbb{N}$ such that $H_{\epsilon, 2}^{t_{U}}(U)$ contains a similar polygon that intersects two discontinuity lines.

Proof. The Claim follows immediately from the following basic properties, using also that $\mathcal{M}_{\epsilon}$ has finite area. Let $U \subset \mathcal{M}_{\epsilon}$ be a convex polygon with boundaries parallel to discontinuity lines. One of the following case applies

- either the interior of $U$ is included in a single atom,

- or the interior intersects a single discontinuity line,

- or it intersects two discontinuity lines.

If the interior of $U$ is included in a single atom, then $H_{\epsilon, 2}(U) \subset \mathcal{M}_{\epsilon}$ is a convex polygon with boundaries parallel to discontinuity lines (and strictly larger area).

If the interior of $U$ intersects a discontinuity line, then $H_{\epsilon, 2}(U) \subset \mathcal{M}_{\epsilon}$ consists of the union of two convex polygons with boundaries parallel to discontinuity lines; and one polygon at least has area larger than $U$.

The geometric properties above are easy to establish (details left to the reader). As for the area consideration in the last claim, using that the largest piece the discontinuity line splits $U$ into must have area larger than $\frac{1}{2}|U|$; we deduce that its image must have area larger than $\frac{(2(1-\epsilon))^{2}}{2}|U|$ and $\frac{(2(1-\epsilon))^{2}}{2}>1$ for every $\epsilon \in\left(0,1-\frac{\sqrt{2}}{2}\right)$.

In the case the two discontinuity lines that a polygon of $H_{\epsilon, 2}^{t_{U}}(U)$ intersects are inclined, Fig. 12 top indicates that this polygon must also intersect $\left\{\frac{1}{2}\right\} \times \mathbb{T}^{1}$ and we are done. Otherwise, ${ }^{19}$ the fact

\footnotetext{
${ }^{18} \mathrm{An}$ atom is defined to be a connected component on which $H_{\epsilon, 2}$ is continuous.

${ }^{19}$ In that case, $H_{\epsilon, 2}^{t_{U}}(U)$ must contain a convex polygon that intersect one horizontal and one inclined discontinuity lines.
} 
that every edge must be parallel to a discontinuity line implies that the intersection with the two discontinuity lines must contain a segment - and indeed a strip - parallel to either the diagonal or the anti-diagonal. In this case, the Lemma follows from the claim below, together with symmetry considerations. ${ }^{20}$ By inclined strip, we mean a strip having two edges parallel to the diagonal.

Claim B.15 Consider any inclined strip $U$ lying across $P_{2, \epsilon}$ (resp. $\left.P_{5, \epsilon}\right)$. This means that an edge is contained in an horizontal discontinuity line and another edge is contained in discontinuity line parallel to the anti-diagonal. Then, there exists $t_{U} \in \mathbb{N}$ such that $H_{\epsilon, 2}^{t_{U}}(U)$ contains an open ball that intersects $\left\{\frac{1}{2}\right\} \times \mathbb{T}^{1}$.

Proof. First consider the case $U$ lies across $P_{2, \epsilon}$. Then $H_{\epsilon, 2}(U)$ is an inclined strip lying either across $S_{v}\left(P_{2, \epsilon}\right)$ or across $S_{v}\left(P_{3, \epsilon}\right)$ (see Fig. 12). In the first case, $H_{\epsilon, 2}^{2}(U)$ contains an inclined strip across $P_{2, \epsilon}$ which is further away than $U$ from the vertical axis $\{0(\bmod 1)\} \times \mathbb{T}^{1}$ and has horizontal width $2(1-\epsilon)$ times larger.

Repeating the argument, we conclude that for any inclined strip $U$ across $P_{2, \epsilon}$, there exists $t \in \mathbb{N}$ such that $H_{\epsilon, 2}^{t}(U)$ contains a $(2(1-\epsilon))^{t}$ broader inclined strip across $S_{v}\left(P_{3, \epsilon}\right)$.

Now, an inclined strip across $S_{v}\left(P_{3, \epsilon}\right)$ is mapped onto an inclined strip lying across the union $S_{u}\left(P_{4, \epsilon} \cup P_{5, \epsilon}\right)$. However, the image of any inclined strip lying across (the extended part of) $S_{u}\left(P_{4, \epsilon}\right)$ intersects $\left\{\frac{1}{2}\right\} \times \mathbb{T}^{1}$; hence we only have to consider the case of an inclined strip across $S_{u}\left(P_{5, \epsilon}\right)$ that hits its bottom horizontal edge.

It results from the definition of $H_{2, \epsilon}$ that the iterate of such strip must contain an inclined strip across $P_{2, \epsilon}$. From the argument above, it follows that a further iterate must eventually cover a broader strip across $S_{u}\left(P_{4, \epsilon} \cup P_{5, \epsilon}\right)$ with larger horizontal width. Hence, some iterate must eventually intersects the line $\left\{\frac{1}{2}\right\} \times \mathbb{T}^{1}$. This completes the proof in the case $U$ lies across $P_{2, \epsilon}$.

In the case $U$ lies across $P_{5, \epsilon}$, the image $H_{2, \epsilon}(U)$ lies across $S_{u}\left(P_{2, \epsilon} \cup P_{3, \epsilon}\right)$ and reaches their upper horizontal boundary. Therefore, it fits one the symmetric cases that were previously considered. Consequently, some iterate must intersects the vertical axis $\left\{\frac{1}{2}\right\} \times \mathbb{T}^{1}$ and the proof is complete.

\section{B.4 Dynamics in contractive regime, proof of Proposition 4.5}

The analogous picture to Fig. 9 for $\epsilon \in\left(\frac{1}{2}, 1\right)$ shows that

$$
H_{\epsilon, 2}(\mathrm{I}) \subset \mathrm{I}
$$

and $H_{\epsilon, 2}(\mathrm{II})$ may still intersect the same three sets as in Fig. 9. By using symmetries and translational invariance, we conclude that the following alternative must hold for every orbit that never hit a discontinuity

- either the orbit end up entering the set $\mathcal{I}_{1}$ and therefore converges to the stable fixed point $(0,0)$,

- or the orbit switches forever back and forth between II and $S_{v}$ (II) (or between $S_{u}$ (II) and $\left.S_{u} \circ S_{v}(\mathrm{II})\right)$. In this case, it must asymptotically approach the 2-periodic orbit on the axis $\{0(\bmod 1)\} \times \mathbb{T}^{1}$ with components $\left(0, \frac{1}{3}\right)$ and $\left(0, \frac{2}{3}\right)$.

Proposition 4.5 follows immediately when applying the change of variable $M^{-1}$. In particular, the periodic orbit generates 3 distinct periodic orbits in the original variables $\left(y_{1}, y_{2}\right)$.

\footnotetext{
${ }^{20}$ In principle, one should also consider similar strips in $H_{\epsilon, 2} \circ S_{v}\left(P_{3, \epsilon}\right)$ (and symmetric strips). However, the Claim is trivial in this case because the strip itself contains an open ball that intersects $\left\{\frac{1}{2}\right\} \times \mathbb{T}^{1}$.
} 